Working Papers of the University of Vaasa, Department of Mathematics and Statistics, 4

\title{
Q-functions of Hermitian contractions of Krein-Ovcharenko type
}

Yury Arlinkiu, Seppo Hassi, and Henk de Snoo

Preprint, May 2003

University of Vaasa

Department of Mathematics and Statistics

P.O. Box 700, FIN-65101 Vaasa, Finland

Preprints are available at: http://www.uwasa.fi/julkaisu/sis.html 


\title{
Q-FUNCTIONS OF HERMITIAN CONTRACTIONS OF KREIN-OVCHARENKO TYPE
}

\author{
YU.M. ARLINSKII, S. HASSI, AND H.S.V. DE SNOO
}

\begin{abstract}
In this paper operator valued $Q$-functions of Krein-Ovcharenko type are introduced. Such functions arise from the extension theory of Hermitian contractive operators $A$ in a Hilbert space $\mathfrak{H}$. The definition is related to the investigations of M.G. Krĕn and I.E. Ovcharenko on the so-called $Q_{\mu^{-}}$and $Q_{M}$-functions. It turns out that their characterizations of such functions hold true only in the matrix valued case. The present paper extends the corresponding properties for wider classes of selfadjoint contractive extensions of $A$. For this purpose some peculiar but fundamental properties on the behaviour of operator ranges of positive operators will be used. Also proper characterizations for $Q_{\mu^{-}}$and $Q_{M}$-functions in the general operator valued case are given. Shorted operators and parallel sums of positive operators will be needed to give a geometric understanding of the function theoretic properties of the corresponding $Q$-functions.
\end{abstract}

\section{INTRODUCTION}

The extension theory of Hermitian contractive and nonnegative operators was established by M.G. Kreln in his famous paper [24]. Subsequently several applications of this theory for solving various problems in mathematical analysis have been given and the theory has been further developed and extended for more general spaces and wider classes of operators.

Let $A$ be a Hermitian contraction in a Hilbert space $\mathfrak{H}$ denined on a subspace $\operatorname{dom} A$ of $\mathfrak{H}$. One of the fundamental results in [24] was the description of all selfadjoint contractive (sc) extensions of $A$ as an operator interval $\left[A_{\mu}, A_{M}\right]$. The endpoints $A_{\mu}$ and $A_{M}$ of this operator interval were characterized by using shorted operators, a notion that was introduced also in [24] (without this name), cf. also [1], [4], [3], [21], [28], [29], [34].

Analytical aspects of the extension theory have been studied in the framework of so-called $Q$-functions associated with the selfadjoint extensions $\widetilde{A}$ of a symmetric operator $A$. These functions appear in Kreun's resolvent formula and they characterize the pair $A, \widetilde{A}$ up to unitary equivalence, see [25]. In the case of a nondensely defined Hermitian contraction $A$ M.G. Krein and I.E. Ovcharenko introduced so-called $Q_{\mu^{-}}$and $Q_{M^{-}}$functions of the form

$$
Q_{\mu}(z)=\left[\left(A_{M}-A_{\mu}\right)^{1 / 2}\left(A_{\mu}-z I\right)^{-1}\left(A_{M}-A_{\mu}\right)^{1 / 2}+I\right] \uparrow \mathfrak{N}, \quad z \in \mathbb{C} \backslash[-1,1],
$$

and

$$
Q_{M}(z)=\left[\left(A_{M}-A_{\mu}\right)^{1 / 2}\left(A_{M}-z I\right)^{-1}\left(A_{M}-A_{\mu}\right)^{1 / 2}-I\right]\lceil\mathfrak{N}, \quad z \in \mathbb{C} \backslash[-1,1],
$$

respectively. These functions take values in the class $\mathcal{L}(\mathfrak{N})$ of bounded operators acting on the subspace $\mathfrak{N}=\mathfrak{H} \ominus \operatorname{dom} A$. Moreover, they belong to the class of Nevanlinna functions, i.e.,

1991 Mathematics Subject Classification. Primary: 47A10, 47A56, 47A64; Secondary 47A05, 47A06, $47 \mathrm{~B} 15$.

Key words and phrases. Hermitian contraction, selfadjoint extension, operator interval, extreme point, shorted operator, parallel sum, $Q$-function.

The first author was partially supported by the Academy of Finland (project 79775) and the Research Institute for Technology at the University of Vaasa. 
they are holomorphic on $\mathbb{C} \backslash \mathbb{R}$ and satisfy the relations $Q(z)^{*}=Q(\bar{z})$ and $\operatorname{Im} z \operatorname{Im} Q(z) \geq 0$ for $z \in \mathbb{C} \backslash \mathbb{R}$. In addition, they admit an analytical continuation to Ext $[-1,1]$ and they are connected by $Q_{\mu}(z) Q_{M}(z)=Q_{M}(z) Q_{\mu}(z)=-I \uparrow \mathfrak{N}$. In their paper [26] M.G. Kreln and I.E. Ovcharenko treated the inverse problem for $Q_{\mu^{-}}$and $Q_{M^{-}}$-functions and suggested some analytical characterizations for these functions among the class of Nevanlinna functions holomorphic on Ext $[-1,1]$. Here the limiting behaviour of these functions at $\infty$ and at the points $z=1, z=-1$ plays a fundamental role. If $Q$ is the $Q_{\mu}$-function of some nondensely defined Hermitian contraction $A$, then it follows from the operator representation (1.1) and the extremal properties of the $s c$-extensions $A_{\mu}$ and $A_{M}$ that $Q(z)$ satisfies the following limit conditions:
1) $s-\lim _{z \rightarrow \infty} Q(z)=I$,
2) $\lim _{z \uparrow-1}(Q \rightarrow \infty(z) h, h)=+\infty$, for all $h \in \mathfrak{N} \backslash\{0\}$,
3) $s-\lim _{z \downarrow 1} Q(z)=0$.

One of the principal results in [26], cf. [26, Theorem 2.2], contains the following assertion: if a Nevanlinna function $Q$, holomorphic on Ext $[-1,1]$ and with values in the class $\mathcal{L}(\mathfrak{N})$ has the properties 1 ) -3 ), then there is a Hilbert space $\mathfrak{H}$ extending $\mathfrak{N}$ and a Hermitian contraction $A$ in $\mathfrak{H}$ defined on $\mathfrak{H} \ominus \mathfrak{N}$, such that $Q$ is the $Q_{\mu}$-function of $A$, i.e., it admits an operator representation of the form (1.1). However, it turns out that the proof given in [26] for this statement has a gap when $\operatorname{dim} \mathfrak{N}=\infty$. Therefore, the following questions arise: how to characterize the subclass of Nevanlinna functions determined by the conditions 1) - 3), in particular, is there an operator representation for such Nevanlinna functions which is analogous to (1.1), and what are the correct analytical characterizations of $Q_{\mu^{-}}$and $Q_{M^{-}}$ functions in the general case $\operatorname{dim} \mathfrak{N} \leq \infty$ ?

The purpose of this paper is to give complete answers to the questions possed above. In particular, operator theoretical interpretations for the limit properties 1) -3) are given in the general case $\operatorname{dim} \mathfrak{N} \leq \infty$, and our investigation yields, for instance, the operator representation stated in the next theorem.

Theorem. Let $\mathfrak{N}$ be a separable Hilbert space and let $Q(z)$ be an operator-valued Nevanlinna function, which is holomorphic on Ext $[-1,1]$ and takes values in $\mathcal{L}(\mathfrak{N})$. Assume that $Q(z)$ satisfies the conditions 1$)-3)$. Then there exist a Hilbert space $\mathfrak{H} \supset \mathfrak{N}$, a Hermitian operator $A$ in $\mathfrak{H}$ defined on $\operatorname{dom} A=\mathfrak{H} \ominus \mathfrak{N}$, and sc-extensions $\widetilde{A}_{1}$ and $\widetilde{A}_{2}$ of $A$, such that

(i) $\widetilde{A}_{1} \leq \widetilde{A}_{2}$,

(ii) $\operatorname{ker}\left(\widetilde{A}_{2}-\widetilde{A}_{1}\right)=\operatorname{dom} A$,

(iii) $\operatorname{ran}\left(\widetilde{A}_{2}-\widetilde{A}_{1}\right)^{1 / 2} \cap \operatorname{ran}\left(\widetilde{A}_{1}-A_{\mu}\right)^{1 / 2}=\operatorname{ran}\left(\widetilde{A}_{2}-\widetilde{A}_{1}\right)^{1 / 2} \cap \operatorname{ran}\left(A_{M}-\widetilde{A}_{2}\right)^{1 / 2}=0$, and such that $Q(z)$ has the operator representation

$$
Q(z)=\left[\left(\widetilde{A}_{2}-\widetilde{A}_{1}\right)^{1 / 2}\left(\widetilde{A}_{1}-z I\right)^{-1}\left(\widetilde{A}_{2}-\widetilde{A}_{1}\right)^{1 / 2}+I\right]\lceil\mathfrak{N} .
$$

If in addition $\operatorname{dim} \mathfrak{N}<\infty$, then $\widetilde{A}_{1}=A_{\mu}$ and $\widetilde{A}_{2}=A_{M}$, and therefore in this case $Q(z)$ is the $Q_{\mu}$-function of $A$.

This theorem shows that the above mentioned statement of M.G. Krein and I.E. Ovcharenko holds true when $\operatorname{dim} \mathfrak{N}<\infty$. In fact, the properties (i)-(iii) of the $s c$-extensions $\widetilde{A}_{1}$ and $\widetilde{A}_{2}$ of $A$ in the previous theorem are closely connected with limit conditions 1) -3 ) for $Q(z)$, but they are not characteristic properties of the extensions $A_{\mu}$ and $A_{M}$ in the case $\operatorname{dim} \mathfrak{N}=\infty$. To one of the basic objectives in this paper becomes the construction of pairs 
$\left\{\widetilde{A}_{1}, \widetilde{A}_{2}\right\}$ of $s c$-extensions of $A$ which satisfy the properties (i)-(iii), but which in general differ from the pair $\left\{A_{\mu}, A_{M}\right\}$ of the endpoints of the corresponding operator interval. As a consequence one obtains examples of operator valued $Q$-functions of Hermitian contractions which are not $Q_{\mu}$-functions (or $Q_{M}$-functions), but still satisfy all the limit conditions 1) - 3) above. From the properties (i)-(iii) of $\widetilde{A}_{1}$ and $\widetilde{A}_{2}$ the most crucial one is the condition (iii); it is equivalent for $\widetilde{A}_{1}$ to be an extreme point of the operator interval $\left[A_{\mu}, \widetilde{A}_{2}\right]$ and for $\widetilde{A}_{2}$ to be an extreme point of the operator interval $\left[\widetilde{A}_{1}, A_{M}\right]$. Assuming that the completely undetermined case holds, i.e. that $\operatorname{ker}\left(A_{M}-A_{\mu}\right)=\operatorname{dom} A$, the construction of other pairs of $s c$-extensions $\left\{\widetilde{A}_{1}, \widetilde{A}_{2}\right\}$ of $A$ satisfying the properties (i)-(iii) is divided into two cases: either $\left(A_{M}-A_{\mu}\right) \mathfrak{N} \neq \mathfrak{N}$ (i.e. $A_{\mu}$ and $A_{M}$ are disjoint) or $\left(A_{M}-A_{\mu}\right) \mathfrak{N}=\mathfrak{N}$ (i.e. $A_{\mu}$ and $A_{M}$ are transversal). Here the second case is more delicate and the problem is first reduced to the following: construct a pair $\{X, \mathfrak{M}\}$ of a nonnegative selfadjoint contraction $X$ in $\mathfrak{N}$ with ker $X=\{0\}$ and a subspace $\mathfrak{M}$ of $\mathfrak{N}$, which in addition admit the following properties

$$
\operatorname{ran} X^{1 / 2} \cap \mathfrak{M}=\operatorname{ran}(I-X)^{1 / 2} \cap(\mathfrak{N} \ominus \mathfrak{M})=\{0\} .
$$

It is also necessary that the subspaces $\mathfrak{M}$ and $\mathfrak{N} \ominus \mathfrak{M}$ in (1.3) both are infinite-dimensional, since otherwise $\left\{\widetilde{A}_{1}, \widetilde{A}_{2}\right\}=\left\{\widetilde{A}_{\mu}, \widetilde{A}_{M}\right\}$. Finally, to show the existence of a pair $\{X, \mathfrak{M}\}$ certain block operator technique will be used as well as the existence of unbounded selfadjoint operators $R$ and $S$ with the property $\operatorname{dom} R \cap \operatorname{dom} S=\{0\}$, a result which is well known and goes back to J. von Neumann in 1929 (cf. [21]; see also [15] for a simple example of such operators in $L^{2}(0,1)$ ). In fact, a complete description of all nonnegative selfadjoint contractions $X$ satisfying the conditions (1.3) is proved.

The paper is organized as follows. In Section 2 some basic notations and preliminary results concerning nonnegative and contractive operators on a Hilbert space are given. It also contains some additions to the basic properties of parallel sums of nonnegative operators, cf. e.g. [2]. Observe, that the condition (iii) in the above theorem can be reformulated by means of parallel sums as follows

$$
\left(\widetilde{A}_{2}-\widetilde{A}_{1}\right):\left(\widetilde{A}_{1}-A_{\mu}\right)=\left(\widetilde{A}_{2}-\widetilde{A}_{1}\right):\left(A_{M}-\widetilde{A}_{2}\right)=0 .
$$

In Section $3 Q$-functions of Krein-Ovcharenko type are introduced and their limit properties at the points $\infty, 1$, and -1 are studied. In particular, the limit conditions in 1) - 3) are connected with the properties (i)-(iii) of the pair $\left\{\widetilde{A}_{1}, \widetilde{A}_{2}\right\}$ of $s c$-extensions of $A$ as indicated above. In Section 4 the main result states that if $\operatorname{dim} \mathfrak{N}=\infty$ then there are pairs $\left\{\widetilde{A}_{1}, \widetilde{A}_{2}\right\}$ of $s c$-extensions of $A$ which are different from the pair $\left\{A_{\mu}, A_{M}\right\}$, but which still satisfy the properties (i)-(iii) above. This result motivates the definition of the new subclasses $\mathfrak{S}_{\mu}(\mathfrak{N})$ and $\mathfrak{S}_{M}(\mathfrak{N})$ of operator valued Nevanlinna functions on $\mathfrak{N}$. In Section 5 the inverse problems for the subclasses $\mathfrak{S}_{\mu}(\mathfrak{N})$ and $\mathfrak{S}_{M}(\mathfrak{N})$ are solved. In particular, the correct version for [26, Theorem 2.2] is obtained in the general case $\operatorname{dim} \mathfrak{N} \leq \infty$. Section 6 contains the precise characterizations for the $Q_{\mu^{-}}$and $Q_{M}$-functions of M.G. Kreŭn and I.E. Ovcharenko, again in the general case $\operatorname{dim} \mathfrak{N} \leq \infty$. Some consequences of the main results obtained in Sections 3-6 for the selfadjoint contractive extensions of Hermitian contractions $A$ are translated by means of Cayley transforms for the case of nonnegative linear relations $S$ in Section 7. This leads to a consideration of closed sesquilinear forms associated with the nonnegative selfadjoint extensions of $S$. In particular, the existence of pairs $\left\{\widetilde{A}_{1}, \widetilde{A}_{2}\right\}$ of $s c$-extensions of $A$ with the properties in (1.4) is shown to be equivalent to the existence of pairs $\left\{\widetilde{S}_{1}, \widetilde{S}_{2}\right\}$ of nonnegative selfadjoint extensions of $S$, such that the form $\widetilde{S}_{1}[\cdot, \cdot]$ is a 
closed restriction of the form $\widetilde{S}_{2}[\cdot, \cdot]$ and the form $\widetilde{S}_{2}^{-1}[\cdot, \cdot]$ is a closed restriction of the form $\widetilde{S}_{1}^{-1}[\cdot, \cdot]$.

\section{NotATIONS AND PRELIMINARIES}

2.1. Basic notations. The symbols $\operatorname{dom} T, \operatorname{ran} T, \operatorname{ker} T$ stand for the domain, range, and null-subspace of a linear operator $T$, and the corresponding closures are denoted by $\overline{\operatorname{dom}} T$, $\overline{\operatorname{ran}} T$. The set of all continuous linear operators defined on a complex Hilbert space $\mathfrak{H}_{1}$ taking values in a complex Hilbert space $\mathfrak{H}_{2}$, is denoted by $\mathcal{L}\left(\mathfrak{H}_{1}, \mathfrak{H}_{2}\right)$, and in the case $\mathfrak{H}=\mathfrak{H}_{1}=\mathfrak{H}_{2}$ shortly by $\mathcal{L}(\mathfrak{H}):=\mathcal{L}(\mathfrak{H}, \mathfrak{H})$. For a closed operator $T$ in $\mathfrak{H}$ the symbol $\rho(T)$ denotes the set of all regular points of $T$. The Moore-Penrose inverse of a bounded selfadjoint operator $B$ is defined by $B^{(-1)}=\left(B\lceil\overline{\operatorname{ran}} B)^{-1} \oplus 0_{\operatorname{ker} B}\right.$.

2.2. Bounded nonnegative operators. Let $\mathfrak{H}$ be a separable Hilbert space. A bounded operator $B$ in $\mathfrak{H}$ is called nonnegative if $(B f, f) \geq 0$ for all $f \in \mathfrak{H}$. If $B$ and $C$ are two bounded selfadjoint operators acting on $\mathfrak{H}$ then the notation $B \geq C$ means that the operator $B-C$ is nonnegative. As is well known the square root $B^{1 / 2}$ of a bounded nonnegative operator $B$ has the following properties:

$$
\begin{gathered}
\operatorname{ran} B^{1 / 2}=\left\{g \in \mathfrak{H}: \sup _{f \in \mathfrak{H}} \frac{|(f, g)|^{2}}{(B f, f)}<\infty\right\}, \\
\left\|B^{(-1 / 2)} g\right\|^{2}=\sup _{f \in \mathfrak{H}} \frac{|(f, g)|^{2}}{(B f, f)}, \quad g \in \operatorname{ran} B^{1 / 2}, \\
\lim _{z \uparrow 0}\left((B-z I)^{-1} g, g\right)= \begin{cases}\left\|B^{(-1 / 2)} g\right\|^{2}, & g \in \operatorname{ran} B^{1 / 2}, \\
+\infty, & g \in \mathfrak{H} \backslash \operatorname{ran} B^{1 / 2},\end{cases}
\end{gathered}
$$

cf. [26].

Lemma 2.1. [19] For every $A, B \in \mathcal{L}(\mathfrak{H})$ the following statements are equivalent:

(i) $\operatorname{ran} A \subset \operatorname{ran} B$;

(ii) $A=B C$ for some $C \in \mathcal{L}(\mathfrak{H})$;

(iii) $A A^{*} \leq \lambda B B^{*}$ for some $\lambda \geq 0$.

In this case there is a unique $C$ satisfying $\operatorname{ker} C=\operatorname{ker} A, \operatorname{ran} C \subset \overline{\operatorname{ran}} B^{*}$, and $\|C\|^{2}=$ $\inf \left\{\lambda: A A^{*} \leq \lambda B B^{*}\right\}$.

Corollary 2.2. For nonnegative $A, B \in \mathcal{L}(\mathfrak{H})$ the following statements are equivalent:

(i) $A \leq B$;

(ii) $A^{1 / 2}=B^{1 / 2} C$ for some contraction $C \in \mathcal{L}(\mathfrak{H})$;

(iii) $A=B^{1 / 2} M B^{1 / 2}$ for some nonnegative contraction $M$ with $\operatorname{ran} M \subset \overline{\operatorname{ran}} B$.

Moreover, in (iii) $M$ is an orthogonal projection if and only if the operator $B^{(-1 / 2)} A^{1 / 2}$ is partial isometry with the initial space $\overline{\operatorname{ran}} A$.

Proof. The equivalence of (i)-(iii) is well known. As to the last statement, let the operator $C$ be defined by $C=B^{(-1 / 2)} A^{1 / 2}$. If $C$ is a partial isometry then $C C^{*}$ is an orthogonal projection onto $\overline{\operatorname{ran}} C \subset \overline{\operatorname{ran}} B$ and $A^{1 / 2}=B^{1 / 2} C$, which gives (iii) with $M=C C^{*}$. Conversely, assume that in (iii) $M$ is an orthogonal projection with $\operatorname{ran} M \subset \overline{\operatorname{ran}} B$. Then $A^{1 / 2}=B^{1 / 2} C$ and $A=B^{1 / 2} C C^{*} B^{1 / 2}$, where $\overline{\operatorname{ran}} C \subset \overline{\operatorname{ran}} B$. Hence, $M=C C^{*}$ by (iii), which means that $C^{*}$ and therefore also $C$ is a partial isometry. By the definition of $C$ its initial space is $\overline{\operatorname{ran}} A$. 
It follows from (iii) in Corollary 2.2 that

$$
\operatorname{ran} A^{1 / 2}=B^{1 / 2}\left(\operatorname{ran} M^{1 / 2}\right) .
$$

Let $\mathfrak{H}=\mathfrak{H}_{1} \oplus \mathfrak{H}_{2}$ be an orthogonal decomposition of $\mathfrak{H}$ and let $B$ be a bounded selfadjoint operator decomposed accordingly, so that $B=\left[B_{i j}\right]_{i, j=1}^{2}$, where $B_{i j}: \mathfrak{H}_{j} \rightarrow \mathfrak{H}_{i}, i, j=1,2$, and $B_{11}^{*}=B_{11}, B_{22}^{*}=B_{22}, B_{21}=B_{12}^{*}$. According to the generalized Sylvester's criterion the operator $B$ is nonnegative if and only if the following conditions are fulfilled:

$$
\text { 1) } \left.B_{11} \geq 0, \quad 2\right) \operatorname{ran} B_{12} \subseteq \operatorname{ran} B_{11}^{1 / 2} \text {, 3) } B_{22} \geq\left(B_{11}^{(-1 / 2)} B_{12}\right)^{*} B_{11}^{(-1 / 2)} B_{12} \text {; }
$$

or equivalently,
1) $B_{11} \geq 0$,
2) $B_{22} \geq 0$
3) $B_{12}=B_{11}^{1 / 2} X B_{22}^{1 / 2}$

where $X: \mathfrak{H}_{2} \rightarrow \mathfrak{H}_{1}$ is a contraction.

2.3. A class of linear relations and related operator ranges. A (closed) linear relation $\tau$ in $\mathfrak{H}$ is a (closed) linear subspace of $\mathfrak{H}^{2}$. The adjoint of $\tau$ is a closed linear relation in $\mathfrak{H}$ given by

$$
\tau^{*}=\left\{\{h, k\} \in \mathfrak{H}^{2}:(k, f)-(h, g)=0 \text { for all }\{f, g\} \in \tau\right\} .
$$

The relation $\tau$ is symmetric if $\tau \subset \tau^{*}$ and selfadjoint if $\tau=\tau^{*}$. Linear relations play an important role in operator theory. In what follows some special classes of symmetric and selfadjoint relations will be useful. Let $A, B \in \mathcal{L}(\mathfrak{H})$ and define (the graph of) the linear relation $\tau$ in $\mathfrak{H}$ by

$$
\tau=\{\{A f, B f\}: f \in \mathfrak{H}\} .
$$

Then the adjoint of $\tau$ takes the form

$$
\tau^{*}=\left\{\{h, k\} \in \mathfrak{H}^{2}: B^{*} h-A^{*} k=0\right\} .
$$

Moreover, $\tau$ is symmetric if and only if $B^{*} A=A^{*} B$ and selfadjoint if and only if

$$
B^{*} A=A^{*} B \text { and } 0 \in \rho(B \pm i A),
$$

cf. [18].

Proposition 2.3. Let the linear relation $\tau$ be defined by $(2.4)$ with $A, B \in \mathcal{L}(\mathfrak{H})$ and let $\mathfrak{L}$, $\mathfrak{M}$ be linear subspaces of $\mathfrak{H}$. Then:

(i) $\operatorname{dom} \tau^{*}=\operatorname{dom} \tau$ if and only if

$$
\operatorname{ran} A=\left\{h \in \mathfrak{H}: B^{*} h \in \operatorname{ran} A^{*}\right\},
$$

in which case $\operatorname{ker} B^{*} \subset \operatorname{ran} A$ and

$$
\operatorname{ran} A \cap \mathfrak{M}=\left(B^{*}\right)^{-1}\left(\operatorname{ran} A^{*}\right) \cap \mathfrak{M} \subset\left(B^{*}\right)^{-1}\left(\operatorname{ran} A^{*} \cap B^{*} \mathfrak{M}\right),
$$

where the last inclusion is an equality if and only if $\operatorname{ker} B^{*} \subset \mathfrak{M}$.

(ii) $\operatorname{ran} \tau^{*}=\operatorname{ran} \tau$ if and only if

$$
\operatorname{ran} B=\left\{k \in \mathfrak{H}: A^{*} k \in \operatorname{ran} B^{*}\right\},
$$

in which case $\operatorname{ker} A^{*} \subset \operatorname{ran} B$ and

$$
\operatorname{ran} B \cap \mathfrak{L}=\left(A^{*}\right)^{-1}\left(\operatorname{ran} B^{*}\right) \cap \mathfrak{L} \subset\left(A^{*}\right)^{-1}\left(\operatorname{ran} B^{*} \cap A^{*} \mathfrak{L}\right),
$$

where the last inclusion is an equality if and only if $\operatorname{ker} A^{*} \subset \mathfrak{L}$.

(iii) If $A^{*} B=B^{*} A$ and one of the conditions in (i) or (ii) is satisfied, then

$$
\operatorname{ran} A^{*} \cap \operatorname{ran} B^{*}=\operatorname{ran} A^{*} B=\operatorname{ran} B^{*} A .
$$


Proof. The equivalences in (i) and (ii) follow easily from the description of $\tau^{*}$ in (2.5). The remaining assertions in (i) and (ii) follow by applying the identities (2.6) and (2.7), respectively.

(iii) Clearly, $\operatorname{ran} A^{*} B=\operatorname{ran} B^{*} A \subset \operatorname{ran} A^{*} \cap \operatorname{ran} B^{*}$. Conversely, assume that $w \in \operatorname{ran} A^{*} \cap$ ran $B^{*}$. Then $w=A^{*} k=B^{*} h$ for some $k, h \in \mathfrak{H}$. In view of (2.5) this means that $\{h, k\} \in \tau^{*}$. Hence, if for instance (i) is satisfied then $h \in \operatorname{dom} \tau=\operatorname{ran} A$, so that $h=A f$ and $w=B^{*} h=B^{*} A f=A^{*} B f$ for some $f \in \mathfrak{H}$. Similarly, from (ii) one obtains $k=B g$ and $w=A^{*} B g=B^{*} A g$ for some $g \in \mathfrak{H}$. This proves the reverse inclusion.

Remark 2.4. (i) Proposition 2.3 can be applied in particular if $\tau$ is selfadjoint. Explicit criteria (allowing infinite-dimensional $\mathfrak{H}$ ) for selfadjointness of a linear relation of the form (2.4), or equivalently of the form (2.5), have been recently obtained in [18].

(ii) If $A^{*} B=B^{*} A, A B^{*}=B A^{*}$, and $A A^{*}+B B^{*}=I$ then $\tau$ need not be selfadjoint (see [18]), but Proposition 2.3 can still be applied. To see this it is enough to prove the inclusions $\operatorname{dom} \tau^{*} \subset \operatorname{dom} \tau$ and $\operatorname{ran} \tau^{*} \subset \operatorname{ran} \tau$, since $A^{*} B=B^{*} A$ means that $\tau \subset \tau^{*}$. Assume that $h \in \operatorname{dom} \tau^{*}$, so that $B^{*} h=A^{*} k$ for some $k \in \mathfrak{H}$. Then $B B^{*} k=B A^{*} k$ which gives $h=A A^{*} h+B A^{*} k=A\left(A^{*} h+B^{*} k\right) \in \operatorname{ran} A=\operatorname{dom} \tau$. Similarly, $k \in \operatorname{ran} \tau^{*}$ implies $k=B\left(B^{*} k+A^{*} h\right) \in \operatorname{ran} B=\operatorname{ran} \tau$. In addition to Proposition 2.3 the given properties of $A$ and $B$ imply that

$$
\operatorname{ran} A \cap \operatorname{ran} B=\operatorname{ran} A B^{*}=\operatorname{ran} B A^{*} .
$$

For this it suffices to prove that $\operatorname{ran} A \cap \operatorname{ran} B \subset \operatorname{ran} B A^{*}$. Now, it $w=A k=B h$ then $A A^{*} A k=A A^{*} B h$ which implies $w=A k=B B^{*} A k+A A^{*} B h=B A^{*}(B k+A h) \in \operatorname{ran} B A^{*}$. In fact, (2.8) reflects the stronger property that the parallel sum of $A A^{*}$ and $B B^{*}$ is equal to $A A^{*}: B B^{*}=\left(A B^{*}\right)^{2}=\left(B A^{*}\right)^{2}$; see (2.18) below.

Let $U \in \mathcal{L}\left(\mathfrak{H}_{1}, \mathfrak{H}_{2}\right)$ be a contraction from the Hilbert space $\mathfrak{H}_{1}$ to the Hilbert space $\mathfrak{H}_{2}$. Then also $U^{*} \in \mathcal{L}\left(\mathfrak{H}_{2}, \mathfrak{H}_{1}\right)$ is a contraction. The defect operator $D_{U}$ associated with $U$ is defined by $D_{U}=\left(I-U^{*} U\right)^{1 / 2}$ and it satisfies the well-known commutation relation, cf. [32]:

$$
U D_{U}=D_{U^{*}} U \text {. }
$$

Corollary 2.5. Let $U \in \mathcal{L}(\mathfrak{H})$ be a contraction, let $D_{U}$ and $D_{U^{*}}$ be the defect operators corresponding to $U$ and $U^{*}$, respectively, and let $\mathfrak{M}$ be a linear subspace of $\mathfrak{H}$. Then:

(i) $\left\{h \in \mathfrak{H}: D_{U^{*}} h \in \operatorname{ran} U\right\}=\operatorname{ran} U$;

(ii) $\operatorname{ran} U \cap \mathfrak{M}=D_{U^{*}}^{-1}(\operatorname{ran} U) \cap \mathfrak{M} \subset D_{U^{*}}^{-1}\left(\operatorname{ran} U \cap D_{U^{*}} \mathfrak{M}\right)$, where the last inclusion is an equality if and only if ker $D_{U^{*}} \subset \mathfrak{M}$;

(iii) $\operatorname{ran} U \cap \operatorname{ran} D_{U^{*}}=\operatorname{ran} U D_{U}=\operatorname{ran} D_{U^{*}} U$.

Proof. Define $A=\left(U U^{*}\right)^{1 / 2}$ and $B=D_{U^{*}}$. Then $A$ and $B$ satisfy the identities

$$
A^{*} B=B^{*} A, \quad A B^{*}=B A^{*}, \quad A^{*} A+B^{*} B=I=A A^{*}+B B^{*} .
$$

It is now easy to check that the pair $A, B$ defines a selfadjoint relation $\tau$ via (2.4); cf. also [18, Proposition 3.7]. Hence, one may apply Proposition 2.3.

(i) The assertion follows from (2.6) and $\operatorname{ran} U=\operatorname{ran}\left(U U^{*}\right)^{1 / 2}=\operatorname{ran} A=\operatorname{ran} A^{*}$ :

$$
\operatorname{ran} U=\operatorname{ran} A=\left\{h \in \mathfrak{H}: D_{U^{*}} h \in \operatorname{ran} A^{*}=\operatorname{ran} U\right\} .
$$

(ii) This follows from part (ii) of Proposition 2.3 and the equalities $\operatorname{ran} U=\operatorname{ran} A=\operatorname{ran} A^{*}$.

(iii) Part (iii) of Proposition 2.3 and the equality $\operatorname{ran} U=\operatorname{ran} A^{*}$ imply that

$$
\operatorname{ran} U \cap \operatorname{ran} D_{U^{*}}=\operatorname{ran}\left(U U^{*}\right)^{1 / 2} D_{U^{*}}=\operatorname{ran}\left(U D_{U}^{2} U^{*}\right)^{1 / 2}=\operatorname{ran} U D_{U}=\operatorname{ran} D_{U^{*}} U,
$$

where the last identity follows from the commutation relation (2.9). 
Corollary 2.6. Let $X \in \mathcal{L}(\mathfrak{H})$ be a nonnegative contraction and let $\mathfrak{M}$ be a linear subspace of $\mathfrak{H}$. Then:

(i) $\left\{h \in \mathfrak{H}:(I-X)^{1 / 2} h \in \operatorname{ran} X^{1 / 2}\right\}=\operatorname{ran} X^{1 / 2}$;

(ii) $\operatorname{ran} X^{1 / 2} \cap \mathfrak{M}=(I-X)^{-1 / 2}\left(\operatorname{ran} X^{1 / 2}\right) \cap \mathfrak{M} \subset(I-X)^{-1 / 2}\left(\operatorname{ran} X^{1 / 2} \cap(I-X)^{1 / 2} \mathfrak{M}\right)$, where the last inclusion is an equality if and only if $\operatorname{ker}(I-X) \subset \mathfrak{M}$;

(iii) $\operatorname{ran} X^{1 / 2} \cap \operatorname{ran}(I-X)^{1 / 2}=\operatorname{ran}\left(X-X^{2}\right)^{1 / 2}$.

Proof. The result is obtained by applying Corollary 2.5 to the nonnegative contraction $U=$ $X^{1 / 2} \in \mathcal{L}(\mathfrak{H})$.

The following statement is of general nature and closely related to part (iii) of Corollary 2.6. Proposition 2.7. For every linear operator $M$ and linear subspace $\mathcal{L}$ one has

$$
M \mathcal{L} \cap(I-M) \mathcal{L} \subset M(I-M) \mathcal{L} .
$$

Moreover, $\operatorname{ran} M \cap \operatorname{ran}(I-M)=\operatorname{ran} M(I-M)$.

Proof. If $y=M u=(I-M) v$ then $M(I-M)(u+v)=(I-M) y+M y=y$, which proves (2.10). The second statement is a consequence of (2.10) and the inclusion $\operatorname{ran} M(I-M) \subset$ $\operatorname{ran} M \cap \operatorname{ran}(I-M)$.

2.4. Shorted operators, parallel addition, and extreme point of operator intervals. For every nonnegative bounded operator $B$ in $\mathfrak{H}$ and every subspace $\mathfrak{N} \subset \mathfrak{H}$, M.G. Kreŭn [24] defined the operator $B_{\mathfrak{N}}$ by the formula

$$
B_{\mathfrak{N}}=\max \{X \in \mathcal{L}(\mathfrak{H}): 0 \leq X \leq B, \operatorname{ran} X \subseteq \mathfrak{N}\}
$$

and established the main properties of $B_{\mathfrak{N}}$, cf. also [1], [2], [3], [21], [28], [34]. In [2] the operator $B_{\mathfrak{N}}$ was called a shorted operator. It follows from Corollary 2.2 that

$$
B_{\mathfrak{N}}=B^{1 / 2} P_{\Omega} B^{1 / 2}
$$

where $P_{\Omega}$ is the orthogonal projection in $\mathfrak{H}$ onto the subspace

$$
\Omega=\left\{f \in \overline{\operatorname{ran}} B: B^{1 / 2} f \in \mathfrak{N}\right\}=\overline{\operatorname{ran}} B \cap B^{-1 / 2} \mathfrak{N} .
$$

Observe, that

$$
\operatorname{ran} B_{\mathfrak{N}}^{1 / 2}=\operatorname{ran} B^{1 / 2} P_{\Omega}=\operatorname{ran} B^{1 / 2} \cap \mathfrak{N}
$$

which shows that

$$
B_{\mathfrak{N}}=0 \text { if and only if } \operatorname{ran} B^{1 / 2} \cap \mathfrak{N}=\{0\} .
$$

The following characterization of $B_{\mathfrak{N}}$ is due to M.G. Krĕ̌n:

$$
\left(B_{\mathfrak{N}} f, f\right)=\inf \{(B(f-\varphi), f-\varphi): \varphi \in \mathfrak{H} \ominus \mathfrak{N}\}, \quad f \in \mathfrak{H} .
$$

The identity (2.11) implies that

$$
B_{\mathfrak{N}}^{1 / 2}=Z B^{1 / 2}=B^{1 / 2} Z^{*}, \quad Z \uparrow \Omega \text { isometric, }
$$

with $Z(\Omega)=\overline{\operatorname{ran}} B_{\mathfrak{N}}^{1 / 2}$ and $Z^{*} Z=P_{\Omega}$, cf. Corollary 2.2.

Let $F$ and $G$ be two bounded nonnegative operators in $\mathfrak{H}$. The parallel sum $F: G$ of $F$ and $G$ is defined as follows

$$
((F: G) h, h):=\inf \{(F f, f)+(G g, g): h=f+g\},
$$

cf. [2], [21], [27]. The following equivalent definition for $F: G$ is easily established (cf. [3], $[30])$

$$
F: G=s-\lim _{\varepsilon \downarrow 0} F(F+G+\varepsilon I)^{-1} G .
$$


Observe, that for every $S \in \mathcal{L}(\mathfrak{H})$ the limit

$$
P_{S}:=s-\lim _{\varepsilon \downarrow 0} S\left(S^{*} S+\varepsilon I\right)^{-1} S^{*}=s-\lim _{\varepsilon \downarrow 0}\left(S S^{*}+\varepsilon I\right)^{-1} S S^{*}
$$

exists and is equal to the orthogonal projection in $\mathfrak{H}$ onto $\overline{\operatorname{ran}} S$. Since $F \leq F+G$, Corollary 2.2 implies that

$$
F=(F+G)^{1 / 2} M(F+G)^{1 / 2}, \quad G=(F+G)^{1 / 2}(I-M)(F+G)^{1 / 2}
$$

for some nonnegative contraction $M$ in $\mathfrak{H}$ with $\operatorname{ran} M \subset \overline{\operatorname{ran}}(F+G)$. This yields another description for $F: G$.

Lemma 2.8. [7] Let the operators $F, G \in \mathcal{L}(\mathfrak{H})$ be nonnegative and let $M$ be as in (2.20). Then:

$$
F: G=(F+G)^{1 / 2}\left(M-M^{2}\right)(F+G)^{1 / 2} .
$$

In particular, $F: G=0$ if and only if $M$ is an orthogonal projection in $\overline{\operatorname{ran}}(F+G)$.

Proof. The representation (2.21) follows from (2.18) and the identity

$$
F(F+G+\varepsilon)^{-1} G=(F+G)^{1 / 2} M(F+G)^{1 / 2}(F+G+\varepsilon)^{-1}(F+G)^{1 / 2}(I-M)(F+G)^{1 / 2},
$$

since $P_{F+G}=s-\lim _{\varepsilon \downarrow 0}(F+G)^{1 / 2}(F+G+\varepsilon)^{-1}(F+G)^{1 / 2}$ is the orthogonal projection onto $\overline{\operatorname{ran}}(F+G)$ by $(2.19)$ and $\operatorname{ran} M \subset \overline{\operatorname{ran}}(F+G)$.

The inclusion $\operatorname{ran} M \subset \overline{\operatorname{ran}}(F+G)$ also guarantees that $F: G=0$ if and only if in $(2.21)$ $M-M^{2}=0$, i.e., $M$ is an orthogonal projection with $\operatorname{ran} M \subset \overline{\operatorname{ran}}(F+G)$.

It is now easy to derive the main properties for parallel sums, cf. e.g. [3], [21]; possibly a new fact here is the necessary and sufficient condition for the equality (2.22). For completeness some short arguments for the other statements are also given.

Proposition 2.9. Let the operators $E, F, G, H \in \mathcal{L}(\mathfrak{H})$ be nonnegative and let $M$ be as in (2.20). Then:

(i) $F: G=G: F$ and $(E: F): G=E:(F: G)$;

(ii) $F: G \leq F$ and $F: G+E: H \leq(F+E):(G+H)$;

(iii) $\operatorname{ran} F \cap \operatorname{ran} G \subset \operatorname{ran}(F: G)$ and $\operatorname{ran}(F: G)^{1 / 2}=\operatorname{ran} F^{1 / 2} \cap \operatorname{ran} G^{1 / 2}$;

(iv) $T(F: G) T^{*} \leq\left(T F T^{*}: T G T^{*}\right)$ for every $T \in \mathcal{L}(\mathfrak{H})$ and here the equality

$$
T(F: G) T^{*}=\left(T F T^{*}: T G T^{*}\right)
$$

holds if and only if the subspace $\overline{\mathrm{ran}}(F+G)^{1 / 2} T^{*}$ is $M$-invariant.

Proof. The properties in (i)-(ii) follow easily from the definition (2.17) of parallel sum.

For the first statement in (iii), observe that if $w=F k=G h$, then (2.20) implies that $y=M(F+G)^{1 / 2} k=(I-M)(F+G)^{1 / 2} h$. Now apply (2.10) to conclude that $y \in \operatorname{ran} M(I-$ $M)(F+G)^{1 / 2}$, so that $w \in \operatorname{ran}(F: G)$.

As to the second statement in (iii), the inclusion $\operatorname{ran}(F: G)^{1 / 2} \subset \operatorname{ran} F^{1 / 2} \cap \operatorname{ran} G^{1 / 2}$ is immediate from Lemma 2.8. Conversely, if $w \in \operatorname{ran} F^{1 / 2} \cap \operatorname{ran} G^{1 / 2}$ then $w=(F+$ $G)^{1 / 2} M^{1 / 2} k=(F+G)^{1 / 2}(I-M)^{1 / 2} h$ and here one may assume that $h \in \overline{\operatorname{ran}}(F+G)$, since $(I-M)^{1 / 2} x=x$ for every $x \in \operatorname{ker}(F+G)$. Then $M^{1 / 2} k=(I-M)^{1 / 2} h$ belongs to $\operatorname{ran} M^{1 / 2}(I-M)^{1 / 2}$ by Corollary 2.6 and Lemma 2.8 gives $w \in \operatorname{ran}(F: G)^{1 / 2}$.

(iv) According to (2.19),

$$
P_{0}:=s-\lim _{\varepsilon \downarrow 0}(F+G)^{1 / 2} T^{*}\left(T(F+G) T^{*}+\varepsilon\right)^{-1} T(F+G)^{1 / 2}
$$


is the orthogonal projection in $\mathfrak{H}$ onto $\overline{\mathrm{ran}}(F+G)^{1 / 2} T^{*}$. Now, write $\left(T F T^{*}: T G T^{*}\right)$ as the strong limit (2.18) by using the expressions (2.20) for $F$ and $G$. Then (2.23) gives

$$
\begin{aligned}
\left(\text { TFT }^{*}: T G T^{*}\right) & =T(F+G)^{1 / 2} M P_{0}(I-M)(F+G)^{1 / 2} T^{*} \\
& =T(F+G)^{1 / 2} P_{0} M P_{0}(I-M) P_{0}(F+G)^{1 / 2} T^{*} .
\end{aligned}
$$

Since $M P_{0} M \leq M^{2},(2.24)$ together with Lemma 2.8 implies that

$$
\left(T F T^{*}: T G T^{*}\right) \geq T(F+G)^{1 / 2} P_{0} M(I-M) P_{0}(F+G)^{1 / 2} T^{*}=T(F: G) T^{*} .
$$

In view of (2.24) and (2.25) the equality (2.22) holds if and only if $P_{0} M P_{0}(I-M) P_{0}=$ $P_{0} M(I-M) P_{0}$, or equivalently, $P_{0} M\left(I-P_{0}\right) M P_{0}=0$. The last condition is equivalent to $M P_{0}=P_{0} M P_{0}$, i.e., the invariance of $\overline{\operatorname{ran}}(F+G)^{1 / 2} T^{*}$ under $M$.

Remark 2.10. If $B=\left[B_{i j}\right]_{i, j=1}^{2} \geq 0$ is decomposed according to $\mathfrak{H}=(\mathfrak{H} \ominus \mathfrak{N}) \oplus \mathfrak{N}$, then

$$
B_{\mathfrak{N}}=\left[\begin{array}{cc}
0 & 0 \\
0 & B_{22}-\left(B_{11}^{(-1 / 2)} B_{12}\right)^{*} B_{11}^{(-1 / 2)} B_{12}
\end{array}\right],
$$

cf. [26]. The parallel sum can be defined also via shorted operators as follows

$$
F: G=\left[\begin{array}{cc}
F+G & F \\
F & F
\end{array}\right]_{0 \oplus \mathfrak{H}}
$$

Some of the results in Subsections 2.3 and 2.4 can be verified also by means of block representations of $B_{\mathfrak{N}}$ and $F: G$ with straightforward calculations.

Observe, that $2(F: G)$ is the harmonic mean of the nonnegative operators $F$ and $G$. Lemma 2.8 and some of the statements in Proposition 2.9 can be extended for general operator means and connections, cf. [27], [7].

The equivalence of the conditions (i), (iii), and (iv) in the next lemma can be found in [20], [29]. In what follows this lemma has a central role; therefore a complete proof, based on Lemma 2.8 , is presented.

Lemma 2.11. (Cf. [20], [29]) Let $[B, C], B \leq C$, be an operator interval in $\mathfrak{H}$. Then $X \in[B, C]$ if and only if

$$
X=B+(C-B)^{1 / 2} M(C-B)^{1 / 2}
$$

for some nonnegative contraction $M$ with $\operatorname{ran} M \subset \overline{\operatorname{ran}}(C-B)$. Moreover, the following conditions for $X \in[B, C]$ are equivalent:

(i) $X=B+(C-B)^{1 / 2} P(C-B)^{1 / 2}$, where $P$ is an orthogonal projection with $\operatorname{ran} P \subset$ $\overline{\operatorname{ran}}(C-B)$;

(ii) $(C-B)^{(-1 / 2)}(X-B)^{1 / 2}$ is a partial isometry with the initial space $\overline{\operatorname{ran}}(X-B)$;

(iii) $(X-B):(C-X)=0$;

(iv) $X$ is an extreme point of $[B, C]$.

Proof. The first statement and the equivalence of (i) and (iii) is obtained by applying Lemma 2.8 with $F=(X-B)$ and $G=C-X$.

By Corollary 2.2 the statements (i) and (ii) are equivalent.

(i), (iii) $\Longrightarrow$ (iv): Let $X$ be as in (i) and assume that $X=\alpha X_{1}+(1-\alpha) X_{2}$ for some $X_{1}, X_{2} \in[B, C]$ and $0<\alpha<1$. Then by part (ii) of Proposition 2.9,

$$
0=(X-B):(C-X) \geq \alpha\left[\left(X_{1}-B\right):\left(C-X_{1}\right)\right]+(1-\alpha)\left[\left(X_{2}-B\right):\left(C-X_{2}\right)\right],
$$


so that $\left(X_{1}-B\right):\left(C-X_{1}\right)=\left(X_{2}-B\right):\left(C-X_{2}\right)=0$. Therefore, $X_{1}$ and $X_{2}$ are of the form (2.28) for some orthogonal projections $P_{1}$ and $P_{2}$ with $\operatorname{ran} P_{j} \subset \overline{\operatorname{ran}}(C-B)$. The assumption now implies that

$$
P=\alpha P_{1}+(1-\alpha) P_{2},
$$

which clearly forces $P=P_{1}=P_{2}$. Thus, $X$ is an extreme point of $[B, C]$.

(iv) $\Longrightarrow$ (iii): Assume that $(X-B):(C-X) \neq 0, X \in[B, C]$. Define $X_{1}=X+(X-B)$ : $(C-X)$ and $X_{2}=X-(X-B):(C-X)$. Then $X_{1}, X_{2} \in[B, C]$ by the first inequality in part (ii) of Proposition. Moreover, $2 X=X_{1}+X_{2}$ and therefore $X$ cannot be an extreme point of $[B, C]$.

Finally, recall the following characterization of $B_{\mathfrak{N}}$ (see [3]):

$$
B_{\mathfrak{N}}=s-\lim _{t \rightarrow+\infty}\left(B: t P_{\mathfrak{N}}\right)
$$

where $P_{\mathfrak{N}}$ is the orthogonal projection in $\mathfrak{H}$ onto $\mathfrak{N}$. It follows from (i) in Proposition 2.9 and (2.29) that

$$
(A: B)_{\mathfrak{N}}=s-\lim _{t \rightarrow+\infty}\left(A:\left(B: t P_{\mathfrak{N}}\right)\right)=A: B_{\mathfrak{N}}
$$

and similarly,

$$
(A: B)_{\mathfrak{N}}=A_{\mathfrak{N}}: B=A_{\mathfrak{N}}: B_{\mathfrak{N}}
$$

\section{Two subclasses of Nevanlinna functions}

In this section two subclasses of the class $\mathbf{N}$ of Nevanlinna functions which are holomorphic on Ext $[-1,1]:=\mathbb{C} \backslash[-1,1]$ are introduced. The motivation comes from the study of selfadjoint contractive extensions of Hermitian contractions.

3.1. Nevanlinna functions holomorphic on Ext $[-1,1]$. Let $\mathcal{H}$ be a Hilbert space. An operator function $V(z)$ with values in $\mathcal{L}(\mathcal{H})$ is said to be a Nevanlinna function or an $R$ function (cf. [22]), if $V(z)$ is holomorphic in the upper and the lower half-plane, $V^{*}(z)=$ $V(\bar{z})$, and $\operatorname{Im} z \operatorname{Im} V(z) \geq 0$ for all $z \in \mathbb{C} \backslash \mathbb{R}$. An $\mathcal{L}(\mathcal{H})$-valued Nevanlinna function $V(z)$ is said to belong to the subclass $\mathbf{N}_{\mathcal{H}}[-1,1]$, if it admits a holomorphic continuation to Ext $[-1,1]$ and has the limit value $V(\infty):=s-\lim _{z \rightarrow \infty} V(z) \in \mathcal{L}(\mathcal{H})$. It is known that every function $V(z)$ in $\mathbf{N}_{\mathcal{H}}[-1,1]$ has an integral representation of the form

$$
V(z)=\Gamma+\int_{-1}^{1} \frac{d G(t)}{t-z}
$$

where $\Gamma$ is a bounded selfadjoint operator in $\mathcal{H}$ and $G(t)$ is a nondecreasing and nonnegative operator function on $[-1,1]$ with values in $\mathcal{H}$, with a finite total variation, and normalized by $G(-1)=0$, cf. [22], [14]. Clearly, $V(\infty)=\Gamma$. The following operator representation for functions $V(z)$ in $\mathbf{N}_{\mathcal{H}}[-1,1]$ is well known.

Theorem 3.1. [14] Let $\mathcal{H}$ be a Hilbert space and let $V(z) \in \mathbf{N}_{\mathcal{H}}[-1,1]$. Then there exist a Hilbert space $\mathfrak{H}$, a selfadjoint contraction $B$ in $\mathfrak{H}$, and an operator $F \in \mathcal{L}(\mathcal{H}, \mathfrak{H})$, such that

$$
V(z)=V(\infty)+F^{*}(B-z I)^{-1} F, \quad z \in \operatorname{Ext}[-1,1] .
$$

Moreover, the representation (3.1) can be chosen to be minimal in the sense that

$$
\overline{\operatorname{span}}\left\{(B-z I)^{-1} F:|z|>1\right\}=\mathfrak{H} \text {. }
$$


When the representation (3.1) is minimal it is uniquely determined in the sense that two such representations with $B \in L(\mathfrak{H}), F \in L(\mathcal{H}, \mathfrak{H})$ and $\widetilde{B} \in L(\mathfrak{K}), \widetilde{F} \in L(\mathcal{H}, \mathfrak{K})$ of $V(z)$ are connected by a unitary operator $\mathcal{U}$ from $\mathfrak{H}$ onto $\mathfrak{K}$ such that the following equalities are satisfied $\widetilde{F}=\mathcal{U} F, \quad \mathcal{U} B=\widetilde{B} \mathcal{U}$.

In this paper functions in the class $\mathbf{N}_{\mathcal{H}}[-1,1]$ with the limit value $V(\infty)= \pm I$ have a central role. The following result is a completion to Theorem 3.1. For a function $V(z) \in$ $\mathbf{N}_{\mathcal{H}}[-1,1], V(\infty)= \pm I$, it gives a sufficient condition which guarantees that the function $-V^{-1}(z)$ also belongs to the class $\mathbf{N}_{\mathcal{H}}[-1,1]$; for simplicity the result is formulated for the case $V(\infty)=I$.

Theorem 3.2. Let $V(z) \in \mathbf{N}_{\mathcal{H}}[-1,1]$ and assume that $V(\infty)=I$. Then $-V^{-1}(z) \in$ $\mathbf{N}_{\mathcal{H}}[-1,1]$ if and only if the strong limit $V(1):=s-\lim _{x \downarrow 1} V(x) \in L(\mathcal{H})$ exists and is nonnegative.

Proof. According to Theorem 3.1 the function $V(z)$ has a minimal operator representation of the form

$$
V(z)=I+F^{*}(B-z I)^{-1} F, \quad z \in \operatorname{Ext}[-1,1] .
$$

It is easy to check that for $z \in \rho(B) \cap \rho\left(B+F F^{*}\right)$ the function $-V^{-1}(z)$ has the operator representation

$$
-V^{-1}(z)=-I+F^{*}\left(B+F F^{*}-z I\right)^{-1} F
$$

Now assume that the limit value $V(1)$ exists. By letting $x \downarrow 1$ in (3.2) one conludes from (2.2) that $\operatorname{ran} F \subset \operatorname{ran}(I-B)^{1 / 2}$ and that

$$
\lim _{x \downarrow 1}(V(x) f, f)=\|f\|^{2}-\left\|(I-B)^{(-1 / 2)} F f\right\|^{2} \geq 0, \quad f \in \mathcal{H} .
$$

Hence, the operator $C:=(I-B)^{(-1 / 2)} F$ is contractive and consequently $F F^{*}=(I-$ $B)^{1 / 2} C C^{*}(I-B)^{1 / 2} \leq I-B$. Since clearly $F F^{*} \geq-(I+B)$, the operator $B+F F^{*}$ is a selfadjoint contraction. This implies that $-V^{-1}(z) \in \mathbf{N}_{\mathcal{H}}[-1,1]$.

Conversely, assume that $-V^{-1}(z) \in \mathbf{N}_{\mathcal{H}}[-1,1]$. It follows from $(3.2)$ that $V^{-1}(\infty)=I$. By Theorem $3.1-V^{-1}(z)$ has a minimal operator representation of the form

$$
-V^{-1}(z)=-I+G^{*}(T-z I)^{-1} G, z \in \operatorname{Ext}[-1,1],
$$

where $T$ is a selfadjoint contration in a Hilbert space $\mathfrak{K}, G \in \mathcal{L}(\mathfrak{H}, \mathfrak{K})$, and

$$
\overline{\operatorname{span}}\left\{(T-z I)^{-1} G:|z|>R\right\}=\mathfrak{K}
$$

holds for every $R>1$. Moreover, it is easy to see that the minimality of the representation of $V(z)$ in (3.2) implies that the representation of $-V^{-1}(z)$ in (3.3) is also minimal, or equivalently that

$$
\overline{\operatorname{span}}\left\{\left(B+F F^{*}-z I\right)^{-1} F:|z|>R\right\}=\mathfrak{H}
$$

for $R$ large enough. Hence, the equality $G^{*}(T-z I)^{-1} G=F^{*}\left(B+F F^{*}-z I\right)^{-1} F$ for $|z|>R$ and $R>1$ large enough together with the minimality conditions (3.5) and (3.4) imply that there exists a unitary operator $\mathcal{U}$ from $\mathfrak{H}$ onto $\mathfrak{K}$ such that the equalities

$$
G=\mathcal{U} F, \quad \mathcal{U}\left(B+F F^{*}\right)=T \mathcal{U}
$$

are satisfied. By unitary equivalence $B+F F^{*}$ is a selfadjoint contraction in $\mathfrak{H}$, i.e. $-I \leq$ $B+F F^{*} \leq I$. It follows that $F F^{*} \leq I-B$ and consequently $s-\lim _{x \downarrow 1} V(x) \geq 0$. 
3.2. Nondensely defined contractions and their contractive extensions. Let $A$ be a Hermitian contraction in $\mathfrak{H}$ defined on the subspace $\operatorname{dom} A \subset \mathfrak{H}$, so that $(A f, g)=(f, A g)$ for all $f, g \in \operatorname{dom} A$ and $\|A\| \leq 1$. A description of all selfadjoint contractive (sc-)extensions of $A$ was given by M.G. Kreln [24]. In fact, he showed that all $s c$-extensions of $A$ form an operator interval $\left[A_{\mu}, A_{M}\right]$, where the extensions $A_{\mu}$ and $A_{M}$ can be characterized by

$$
\left(I+A_{\mu}\right)_{\mathfrak{N}}=0, \quad\left(I-A_{M}\right)_{\mathfrak{N}}=0
$$

respectively. Two equivalent descriptions for $\left[A_{\mu}, A_{M}\right]$ are (cf. [24], [26]):

$$
\widetilde{A}=\left(A_{M}+A_{\mu}\right) / 2+\left(A_{M}-A_{\mu}\right)^{1 / 2} Y\left(A_{M}-A_{\mu}\right)^{1 / 2} / 2,
$$

where $Y$ is a $s c$-operator in the subspace $\mathfrak{N}_{0}:=\overline{\operatorname{ran}}\left(A_{M}-A_{\mu}\right) \subseteq \mathfrak{N}$, and

$$
\widetilde{A}=A_{\mu}+\left(A_{M}-A_{\mu}\right)^{1 / 2} X\left(A_{M}-A_{\mu}\right)^{1 / 2}, \quad X \in\left[0, I_{\mathfrak{N}_{0}}\right] .
$$

The connection between (3.7) and (3.8) is via $X=(I+Y) / 2$. It follows from (3.6), for instance by using (2.26), that for every $s c$-extension $\widetilde{A}$ of $A$ the following identities hold:

$$
(I-\widetilde{A})_{\mathfrak{N}}=A_{M}-\widetilde{A}, \quad(I+\widetilde{A})_{\mathfrak{N}}=\widetilde{A}-A_{\mu},
$$

cf. [24]. Hence, according to (2.11)

$$
\operatorname{ran}(I-\widetilde{A})^{1 / 2} \cap \mathfrak{N}=\operatorname{ran}\left(A_{M}-\widetilde{A}\right)^{1 / 2}, \quad \operatorname{ran}(I+\widetilde{A})^{1 / 2} \cap \mathfrak{N}=\operatorname{ran}\left(\widetilde{A}-A_{\mu}\right)^{1 / 2} .
$$

An sc-extension $\widetilde{A}$ of $A$ is said to be an extremal extension of $A$ if $\widetilde{A}$ is an extreme point of the operator interval $\left[A_{\mu}, A_{M}\right]$. The next result contains a simple characterization for the extremality of an $s c$-extension $\widetilde{A}$ of $A$, which is based on shorted operators.

Proposition 3.3. Let $\widetilde{A} \in\left[A_{\mu}, A_{M}\right]$ be an sc-extension of the Hermitian contraction $A$ in $\mathfrak{H}$ and let $\mathfrak{N}=\mathfrak{H} \ominus \operatorname{dom} A$. Then

$$
\left(\widetilde{A}-A_{\mu}\right):\left(A_{M}-\widetilde{A}\right)=\frac{1}{2}\left(I-\widetilde{A}^{2}\right)_{\mathfrak{N}},
$$

and $\operatorname{ran}\left(I-\widetilde{A}^{2}\right)^{1 / 2} \cap \mathfrak{N}=\operatorname{ran}\left(\widetilde{A}-A_{\mu}\right)^{1 / 2} \cap\left(A_{M}-\widetilde{A}\right)^{1 / 2}$. In particular, $\widetilde{A}$ is an extremal extension of $A$ if and only if

$$
\left(I-\widetilde{A}^{2}\right)_{\mathfrak{N}}=0 .
$$

Proof. The equality (3.11) follows from (2.30) and (3.9):

$$
\left(\widetilde{A}-A_{\mu}\right):\left(A_{M}-\widetilde{A}\right)=(I+\widetilde{A})_{\mathfrak{N}}:(I-\widetilde{A})_{\mathfrak{N}}=(I+\widetilde{A}: I-\widetilde{A})_{\mathfrak{N}}=\frac{1}{2}((I+\widetilde{A})(I-\widetilde{A}))_{\mathfrak{N}} .
$$

Here the last identity is obtained e.g. by applying Lemma 2.8 with $M=\frac{1}{2}(I-\widetilde{A})$.

The statement concerning the ranges is clear from (2.13) and (3.11), while the last assertion follows from Lemma 2.11.

Corollary 3.4. Let $\widetilde{A}_{1}, \widetilde{A}_{2} \in\left[A_{\mu}, A_{M}\right]$ with $\widetilde{A}_{1} \leq \widetilde{A}_{2}$ be sc-extensions of the Hermitian contraction $A$ in $\mathfrak{H}$ and let $\mathfrak{N}=\mathfrak{H} \ominus \operatorname{dom} A$.

(i) If $\widetilde{A}_{1}$ is an extreme point of $\left[A_{\mu}, \widetilde{A}_{2}\right]$ then

$$
\left(I-\widetilde{A}_{1}^{2}\right)_{\mathfrak{N}}:\left(\widetilde{A}_{2}-\widetilde{A}_{1}\right)=\left(I-\widetilde{A}_{1}^{2}\right):\left(\widetilde{A}_{2}-\widetilde{A}_{1}\right)=0 ;
$$

(ii) If $\widetilde{A}_{2}$ is an extreme point of $\left[\widetilde{A}_{1}, A_{M}\right]$ then

$$
\left(I-\widetilde{A}_{2}^{2}\right)_{\mathfrak{N}}:\left(\widetilde{A}_{2}-\widetilde{A}_{1}\right)=\left(I-\widetilde{A}_{2}^{2}\right):\left(\widetilde{A}_{2}-\widetilde{A}_{1}\right)=0 .
$$


Proof. By Lemma 2.11 the assumption in (i) is equivalent to $\left(\widetilde{A}_{1}-A_{\mu}\right):\left(\widetilde{A}_{2}-\widetilde{A}_{1}\right)=0$. Hence, it follows from Proposition 3.3

$$
\begin{aligned}
\frac{1}{2}\left(I-\widetilde{A}_{1}^{2}\right)_{\mathfrak{N}}:\left(\widetilde{A}_{2}-\widetilde{A}_{1}\right) & =\left(\left(\widetilde{A}_{1}-A_{\mu}\right):\left(A_{M}-\widetilde{A}_{1}\right)\right):\left(\widetilde{A}_{2}-\widetilde{A}_{1}\right) \\
& =\left(A_{M}-\widetilde{A}_{1}\right):\left(\left(\widetilde{A}_{1}-A_{\mu}\right):\left(\widetilde{A}_{2}-\widetilde{A}_{1}\right)\right)=0 .
\end{aligned}
$$

Moreover, in view of (2.30) one obtains

$$
\left(I-\widetilde{A}_{1}^{2}\right)_{\mathfrak{N}}:\left(\widetilde{A}_{2}-\widetilde{A}_{1}\right)=\left(I-\widetilde{A}_{1}^{2}\right):\left(\widetilde{A}_{2}-\widetilde{A}_{1}\right)_{\mathfrak{N}}=\left(I-\widetilde{A}_{1}^{2}\right):\left(\widetilde{A}_{2}-\widetilde{A}_{1}\right) .
$$

This gives (i) and similarly one proves (ii).

Remark 3.5. The characterization (3.12) in Proposition 3.3 for the extremality of an $s c$ extension $\widetilde{A}$ of $A$ was given in [10]. Here it is an immediate consequence of the equality (3.11).

Using matrix representations the fundamental results due to M.G. Kreln on extensions of contractions were extended to the more general case of contractive extensions of dual pairs of contractions in [35], [11]; for operator representations cf. also [6].

3.3. $Q$-functions of Krein-Ovcharenko type. Let $A$ be a Hermitian contraction in $\mathfrak{H}$ defined on the subspace $\operatorname{dom} A \subset \mathfrak{H}$. In the sequel it is assumed that the completely undetermined case holds, i.e.,

$$
\operatorname{Ker}\left(A_{M}-A_{\mu}\right)=\operatorname{dom} A .
$$

Let $\widetilde{A}_{1}, \widetilde{A}_{2} \in\left[A_{\mu}, A_{M}\right], \widetilde{A}_{1} \leq \widetilde{A}_{2}$, and define on $\mathfrak{N}=\mathfrak{H} \ominus \operatorname{dom} A$ the following operator functions, holomorphic on $\operatorname{Ext}[-1,1]$ :

$$
\begin{aligned}
& \widetilde{Q}_{1}(z)=\left[\left(\widetilde{A}_{2}-\widetilde{A}_{1}\right)^{1 / 2}\left(\widetilde{A}_{1}-z I\right)^{-1}\left(\widetilde{A}_{2}-\widetilde{A}_{1}\right)^{1 / 2}+I\right]\lceil\mathfrak{N}, \\
& \widetilde{Q}_{2}(z)=\left[\left(\widetilde{A}_{2}-\widetilde{A}_{1}\right)^{1 / 2}\left(\widetilde{A}_{2}-z I\right)^{-1}\left(\widetilde{A}_{2}-\widetilde{A}_{1}\right)^{1 / 2}-I\right]\lceil\mathfrak{N},
\end{aligned}
$$

In particular, when $\widetilde{A}_{1}=A_{\mu}$ and $\widetilde{A}_{2}=A_{M}$ one obtains the functions $Q_{\mu}(z)$ and $Q_{M}(z)$ studied by M.G. Kreın and I.E. Ovcharenko in [26]:

$$
\begin{aligned}
& Q_{\mu}(z)=\left[\left(A_{M}-A_{\mu}\right)^{1 / 2}\left(A_{\mu}-z I\right)^{-1}\left(A_{M}-A_{\mu}\right)^{1 / 2}+I\right]\lceil\mathfrak{N}, \\
& Q_{M}(z)=\left[\left(A_{M}-A_{\mu}\right)^{1 / 2}\left(A_{M}-z I\right)^{-1}\left(A_{M}-A_{\mu}\right)^{1 / 2}+I\right]\lceil\mathfrak{N} .
\end{aligned}
$$

Clearly, the functions $\widetilde{Q}_{1}(z)$ and $\widetilde{Q}_{2}(z)$ belong to $\mathbf{N}_{\mathfrak{N}}[-1,1]$. Moreover, it is easy to see that

$$
\widetilde{Q}_{1}(z) \widetilde{Q}_{2}(z)=\widetilde{Q}_{2}(z) \widetilde{Q}_{1}(z)=-I \uparrow \mathfrak{N}, \quad z \in \operatorname{Ext}[-1,1] .
$$

Observe, that

$$
\lim _{z \rightarrow \infty} \widetilde{Q}_{1}(z)=I_{\mathfrak{N}}, \quad \lim _{z \rightarrow \infty} \widetilde{Q}_{2}(z)=-I_{\mathfrak{N}}
$$

Associate with an $s c$-extension $\widetilde{A}$ of $A$ the subspaces

$$
\Omega_{ \pm}(\widetilde{A}):=\left\{f \in \overline{\operatorname{ran}}(I \pm \widetilde{A}):(I \pm \widetilde{A})^{1 / 2} f \in \mathfrak{N}\right\}
$$

cf. (2.12). The next theorem describes the limiting behaviour of $\widetilde{Q}_{1}(z)$ and $\widetilde{Q}_{2}(z)$ at \pm 1 .

Theorem 3.6. The functions $\widetilde{Q}_{1}(z)$ and $\widetilde{Q}_{2}(z)$ defined in (3.14), (3.15) satisfy the following limit formulas: 
(i) $\lim _{z \downarrow 1}\left(\widetilde{Q}_{1}(z) h, h\right)=\|h\|^{2}-\left\|\left(A_{M}-\widetilde{A}_{1}\right)^{(-1 / 2)}\left(\widetilde{A}_{2}-\widetilde{A}_{1}\right)^{1 / 2} h\right\|^{2}, h \in \mathfrak{N}$;

(ii) $\lim _{z \uparrow-1}\left(\widetilde{Q}_{2}(z) h, h\right)=-\|h\|^{2}+\left\|\left(\widetilde{A}_{2}-A_{\mu}\right)^{(-1 / 2)}\left(\widetilde{A}_{2}-\widetilde{A}_{1}\right)^{1 / 2} h\right\|^{2}, h \in \mathfrak{N}$;

(iii) $\lim _{z \uparrow-1}\left(\widetilde{Q}_{1}(z) h, h\right)= \begin{cases}\|h\|^{2}+\left\|\left(\widetilde{A}_{1}-A_{\mu}\right)^{(-1 / 2)}\left(\widetilde{A}_{2}-\widetilde{A}_{1}\right)^{1 / 2} h\right\|^{2}, & h \in \mathfrak{D}_{1}, \\ +\infty, & h \in \mathfrak{N} \backslash \mathfrak{D}_{1} ;\end{cases}$

(iv) $\lim _{z \downarrow 1}\left(\widetilde{Q}_{2}(z) h, h\right)= \begin{cases}-\|h\|^{2}-\left\|\left(A_{M}-\widetilde{A}_{2}\right)^{(-1 / 2)}\left(\widetilde{A}_{2}-\widetilde{A}_{1}\right)^{1 / 2} h\right\|^{2}, & h \in \mathfrak{D}_{2}, \\ -\infty, & h \in \mathfrak{N} \backslash \mathfrak{D}_{2} ;\end{cases}$

where $\mathfrak{D}_{1}=\left\{h \in \mathfrak{N}:\left(\widetilde{A}_{2}-\widetilde{A}_{1}\right)^{1 / 2} h \in \operatorname{ran}\left(\widetilde{A}_{1}-A_{\mu}\right)^{1 / 2}\right\}$ and $\mathfrak{D}_{2}=\left\{h \in \mathfrak{N}:\left(\widetilde{A}_{2}-\widetilde{A}_{1}\right)^{1 / 2} h \in\right.$ $\left.\operatorname{ran}\left(A_{M}-\widetilde{A}_{1}\right)^{1 / 2}\right\}$.

Proof. The inequalities $A_{\mu} \leq \widetilde{A}_{1} \leq \widetilde{A}_{2} \leq A_{M}$ imply that

$$
\operatorname{ran}\left(\widetilde{A}_{2}-\widetilde{A}_{1}\right)^{1 / 2} \subset \operatorname{ran}\left(A_{M}-\widetilde{A}_{1}\right)^{1 / 2} \subset \operatorname{ran}\left(I-\widetilde{A}_{1}\right)^{1 / 2} .
$$

In view of (2.2) and (3.14) one obtains

$$
\lim _{z \downarrow 1}\left(\widetilde{Q}_{1}(z) h, h\right)=\|h\|^{2}-\left\|\left(I-\widetilde{A}_{1}\right)^{(-1 / 2)}\left(\widetilde{A}_{2}-\widetilde{A}_{1}\right)^{1 / 2} h\right\|^{2}, \quad h \in \mathfrak{N} .
$$

Write

$$
\left(I \pm \widetilde{A}_{1}\right)_{\mathfrak{N}}=\left(I \pm \widetilde{A}_{1}\right)^{1 / 2} P_{ \pm}\left(I \pm \widetilde{A}_{1}\right)^{1 / 2}
$$

where $P_{ \pm}$are the orthogonal projections in $\mathfrak{H}$ onto $\Omega_{ \pm}\left(\widetilde{A}_{1}\right)$, cf. (2.11), (3.19). The identities (2.16) and (3.9) show that

$$
\begin{aligned}
\left(A_{M}-\widetilde{A}_{1}\right)^{1 / 2} & =Z_{-} P_{-}\left(I-\widetilde{A}_{1}\right)^{1 / 2}=\left(I-\widetilde{A}_{1}\right)^{1 / 2} Z_{-}^{*}, \\
\left(\widetilde{A}_{1}-A_{\mu}\right)^{1 / 2} & =Z_{+} P_{+}\left(I+\widetilde{A}_{1}\right)^{1 / 2}=\left(I+\widetilde{A}_{1}\right)^{1 / 2} Z_{+}^{*},
\end{aligned}
$$

where $Z_{ \pm}$are isometries from $\Omega_{ \pm}\left(\widetilde{A}_{1}\right)$ into $\mathfrak{H}$. The first relation in (3.21) gives

$$
\left\|\left(I-\widetilde{A}_{1}\right)^{(-1 / 2)}\left(\widetilde{A}_{2}-\widetilde{A}_{1}\right)^{1 / 2} h\right\|^{2}=\left\|\left(A_{M}-\widetilde{A}_{1}\right)^{(-1 / 2)}\left(\widetilde{A}_{2}-\widetilde{A}_{1}\right)^{1 / 2} h\right\|^{2}
$$

for all $h \in \mathfrak{N}$. This proves (i). Similarly one obtains (iii) from (2.2) and the second identity in $(3.21)$ by observing that

$$
\left\{h \in \mathfrak{N}:\left(\widetilde{A}_{2}-\widetilde{A}_{1}\right)^{1 / 2} h \in \operatorname{ran}\left(\widetilde{A}_{1}+I\right)^{1 / 2}\right\}=\mathfrak{D}_{1}
$$

in view of (2.13).

The proofs of (ii) and (iv) are analogous.

When $\widetilde{A}_{1}=A_{\mu}$ and $\widetilde{A}_{2}=A_{M}$ Theorem 3.6 and the assumption (3.13) give the following relations, which were obtained in [26]:

$$
\begin{array}{ll}
s-\lim _{z \downarrow 1} Q_{\mu}(z)=0, & \lim _{z \uparrow-1}\left(Q_{\mu}(z) h, h\right)=+\infty, h \in \mathfrak{N} \backslash\{0\} ; \\
s-\lim _{z \uparrow-1} Q_{M}(z)=0, & \lim _{z \downarrow 1}\left(Q_{M}(z) h, h\right)=-\infty, h \in \mathfrak{N} \backslash\{0\} .
\end{array}
$$

Lemma 3.7. Let $\widetilde{Q}_{1}(z)$ and $\widetilde{Q}_{2}(z)$ be defined by (3.14), (3.15). Then the following conditions are equivalent:

(i) $\lim _{z \uparrow-1}\left(\widetilde{Q}_{1}(z) h, h\right)=+\infty$ for all $h \in \mathfrak{N} \backslash\{0\}$;

(ii) $s-\lim _{z \uparrow-1} \widetilde{Q}_{2}(z)=0$;

(iii) $\left(\widetilde{A}_{1}-A_{\mu}\right):\left(\widetilde{A}_{2}-\widetilde{A}_{1}\right)=0$ and $\operatorname{ker}\left(\widetilde{A}_{2}-\widetilde{A}_{1}\right)=\operatorname{dom} A$; 
(iv) $\widetilde{A}_{1}$ is an extreme point of $\left[A_{\mu}, \widetilde{A}_{2}\right]$ and $\operatorname{ker}\left(\widetilde{A}_{2}-\widetilde{A}_{1}\right)=\operatorname{dom} A$. Moreover, the following conditions are equivalent:

(i') $s-\lim _{z \downarrow 1} \widetilde{Q}_{1}(z)=0$;

(ii') $\lim _{z \downarrow 1}\left(\widetilde{Q}_{2}(z) h, h\right)=-\infty$ for all $h \in \mathfrak{N} \backslash\{0\}$;

(iii') $\left(A_{M}-\widetilde{A}_{2}\right):\left(\widetilde{A}_{2}-\widetilde{A}_{1}\right)=0$ and $\operatorname{ker}\left(\widetilde{A}_{2}-\widetilde{A}_{1}\right)=\operatorname{dom} A$;

(iv') $\widetilde{A}_{2}$ is an extreme point of $\left[\widetilde{A}_{1}, A_{M}\right]$ and $\operatorname{ker}\left(\widetilde{A}_{2}-\widetilde{A}_{1}\right)=\operatorname{dom} A$.

Proof. (i) $\Longleftrightarrow$ (iii) It follows from part (iii) of Theorem 3.6 that the condition (i) is equivalent to $\mathfrak{D}_{1}=\{0\}$, i.e.,

$$
\operatorname{ran}\left(\widetilde{A}_{1}-A_{\mu}\right)^{1 / 2} \cap \operatorname{ran}\left(\widetilde{A}_{2}-\widetilde{A}_{1}\right)^{1 / 2}=\{0\} \quad \text { and } \operatorname{ker}\left(\widetilde{A}_{2}-\widetilde{A}_{1}\right)=\operatorname{dom} A
$$

Now, the equivalence of (i) and (iii) follows now from Proposition 2.9.

(ii) $\Longleftrightarrow$ (iii) $\Longleftrightarrow$ (iv) These equivalences follow immediately from Lemma 2.11.

The second part of the lemma is proved is a similar manner. In particular, the equivalence of (ii') and (iii') is obtained by using the property (iv) in Theorem 3.6.

Corollary 3.8. Let $\widetilde{A}_{1} \leq \widetilde{A}_{2}$ be sc-extensions of $A$ and let $\widetilde{A}_{1}^{\prime}$ and $\widetilde{A}_{2}^{\prime}$ be defined by

$$
\widetilde{A}_{1}^{\prime}=A_{\mu}+A_{M}-\widetilde{A}_{2}, \quad \widetilde{A}_{2}^{\prime}=A_{\mu}+A_{M}-\widetilde{A}_{1} .
$$

Then $\widetilde{A}_{1}^{\prime} \leq \widetilde{A}_{2}^{\prime}$ are also sc-extensions of $A$. Moreover, if the pair $\left\{\widetilde{A}_{1}, \widetilde{A}_{2}\right\}$ satisfies one of the conditions (i)-(iv) in Lemma 3.7, then the pair $\left\{\widetilde{A}_{1}^{\prime}, \widetilde{A}_{2}^{\prime}\right\}$ satisfies the conditions ( $\left.i^{\prime}\right)-(i v ')$ in Lemma 3.7, and if the pair $\left\{\widetilde{A}_{1}, \widetilde{A}_{2}\right\}$ satisfies if one of the conditions ( $\left.i^{\prime}\right)-\left(i v^{\prime}\right)$ in Lemma 3.7, then the pair $\left\{\widetilde{A}_{1}^{\prime}, \widetilde{A}_{2}^{\prime}\right\}$ satisfies the conditions (i)-(iv).

Proof. The first statement is easy to check. Moreover, the other two statements follow from the equalities $\widetilde{A}_{2}^{\prime}-\widetilde{A}_{1}^{\prime}=\widetilde{A}_{2}-\widetilde{A}_{1}, \widetilde{A}_{1}^{\prime}-\widetilde{A}_{\mu}=A_{M}-\widetilde{A}_{2}$, and $A_{M}-\widetilde{A}_{2}^{\prime}=\widetilde{A}_{1}-A_{\mu}$.

Corollary 3.9. Let $\mathfrak{N}=\mathfrak{H} \ominus \operatorname{dom} A$ and assume that $\operatorname{dim} \mathfrak{N}<\infty$. Then:

(i) If one of the conditions (i)-(iv) in Lemma 3.7 is satisfied, then $\widetilde{A}_{1}=A_{\mu}$.

(ii) If one of the conditions ( $\left.{ }^{\prime}\right)^{\prime}-(i v ')$ in Lemma 3.7 is satisfied, then $\widetilde{A}_{2}=A_{M}$.

Proof. (i) If one of the conditions (i)-(iv) in Lemma 3.7 is satisfied, then $\operatorname{ker}\left(\widetilde{A}_{2}-\widetilde{A}_{1}\right)=$ $\operatorname{dom} A$. This implies that $\operatorname{ran}\left(\widetilde{A}_{2}-\widetilde{A}_{1}\right)=\mathfrak{N}$, since $\operatorname{dim} \mathfrak{N}<\infty$. Now, part (iii) of Lemma 3.7 shows that $\operatorname{ran}\left(\widetilde{A}_{1}-\widetilde{A}_{\mu}\right)^{1 / 2} \cap \mathfrak{N}=\{0\}$ and consequently $\widetilde{A}_{1}=A_{\mu}$. This proves (i).

The proof of (ii) is similar.

Corollary 3.9 implies the characterizations obtained by M.G. Kreĭn and I.E. Ovcharenko [26] for the $Q_{\mu}$-functions and $Q_{M}$-functions, in the case that $\operatorname{dim} \mathfrak{N}<\infty$. In the next section it is shown that if $\operatorname{dim} \mathfrak{N}=\infty$, then the limit characterizations (cf. (3.22)) stated in [26] for $Q_{\mu}$-functions and $Q_{M}$-functions fail to hold.

\section{Constructions of SPecial sc-extensions}

In this section a pair $\left\{\widetilde{A}_{1}, \widetilde{A}_{2}\right\}$ of $s c$-extensions is constructed, whose $Q$-functions admit all the limiting properties stated in [26], but which does not coincide with the pair $\left\{A_{\mu}, A_{M}\right\}$ if $\operatorname{dim} \mathfrak{N}=\infty$. By Lemma 3.7 the limiting behaviour of functions appearing in [26] can be translated into conditions involving only the sc-extensions $\widetilde{A}_{1}, \widetilde{A}_{2}, A_{\mu}$, and $A_{M}$. The 
problem can be reformulated as follows: Construct the $s c$-extensions $\widetilde{A}_{1} \leq \widetilde{A}_{2}$ of $A$ with the following properties

$$
\left(\widetilde{A}_{1}-A_{\mu}\right):\left(\widetilde{A}_{2}-\widetilde{A}_{1}\right)=\left(A_{M}-\widetilde{A}_{2}\right):\left(\widetilde{A}_{2}-\widetilde{A}_{1}\right)=0 \text { and } \operatorname{ker}\left(\widetilde{A}_{2}-\widetilde{A}_{1}\right)=\operatorname{dom} A,
$$

and such that pair $\left\{\widetilde{A}_{1}, \widetilde{A}_{2}\right\}$ does not coincide with the pair $\left\{A_{\mu}, A_{M}\right\}$. Again it is assumed that completely undetermined case holds, i.e., equality (3.13) is satisfied. The construction is devided into two cases: $\left(A_{M}-A_{\mu}\right) \mathfrak{N} \neq \mathfrak{N}$ and $\left(A_{M}-A_{\mu}\right) \mathfrak{N}=\mathfrak{N}$.

Remark 4.1. The firts two conditions for the pair $\left\{\widetilde{A}_{1}, \widetilde{A}_{2}\right\}$ of $s c$-extensions of $A$ in $(4.1)$ are can be stated in the following equivalent form:

$$
\left(I+\widetilde{A}_{1}\right):\left(\widetilde{A}_{2}-\widetilde{A}_{1}\right)=\left(I-\widetilde{A}_{2}\right):\left(\widetilde{A}_{2}-\widetilde{A}_{1}\right)=0 .
$$

In fact, applying (2.30) one obtains the following equalities

$$
\left(\widetilde{A}_{1}-A_{\mu}\right):\left(\widetilde{A}_{2}-\widetilde{A}_{1}\right)=\left(I+\widetilde{A}_{1}\right)_{\mathfrak{N}}:\left(\widetilde{A}_{2}-\widetilde{A}_{1}\right)=\left(I+\widetilde{A}_{1}\right):\left(\widetilde{A}_{2}-\widetilde{A}_{1}\right)_{\mathfrak{N}}=\left(I+\widetilde{A}_{1}\right):\left(\widetilde{A}_{2}-\widetilde{A}_{1}\right) \text {. }
$$

Similarly one arrives at the second identity in (4.1).

4.1. The case $\left(A_{M}-A_{\mu}\right) \mathfrak{N} \neq \mathfrak{N}$. Let $\mathfrak{N}_{1} \subset \mathfrak{N}_{2}$ be two subspaces of $\mathfrak{N}$ such that

$$
\mathfrak{N}_{2} \cap\left(A_{M}-A_{\mu}\right)^{1 / 2} \mathfrak{N}=\{0\}
$$

and let $P_{1}$ and $P_{2}$ be orthogonal projections onto $\mathfrak{N}_{1}$ and $\mathfrak{N}_{2}$ in $\mathfrak{N}$, respectively.

Proposition 4.2. Let $\left(A_{M}-A_{\mu}\right) \mathfrak{N} \neq \mathfrak{N}$ and let $L$ be a nonnegative selfadjoint contraction in the subspace $\mathfrak{N}_{1}$, where $\mathfrak{N}_{1} \subset \mathfrak{N}_{2}$, and $\mathfrak{N}_{2}$ satisfies (4.3). Then the sc-extensions of $A$ defined by

$$
\begin{aligned}
& \widetilde{A}_{1}=A_{\mu}+\left(A_{M}-A_{\mu}\right)^{1 / 2} L P_{1}\left(A_{M}-A_{\mu}\right)^{1 / 2} \\
& \widetilde{A}_{2}=A_{\mu}+\left(A_{M}-A_{\mu}\right)^{1 / 2}\left(L P_{1}+\left(I-P_{2}\right)\right)\left(A_{M}-A_{\mu}\right)^{1 / 2}
\end{aligned}
$$

satisfy the equalities (4.1).

Proof. The definitions of $\widetilde{A}_{1}$ and $\widetilde{A}_{2}$ in (4.4) and (3.8) imply

$$
\begin{aligned}
& \widetilde{A}_{2}-\widetilde{A}_{1}=\left(A_{M}-A_{\mu}\right)^{1 / 2}\left(I-P_{2}\right)\left(A_{M}-A_{\mu}\right)^{1 / 2}, \\
& \widetilde{A}_{1}-A_{\mu}=\left(A_{M}-A_{\mu}\right)^{1 / 2} L P_{1}\left(A_{M}-A_{\mu}\right)^{1 / 2}, \\
& A_{M}-\widetilde{A}_{2}=\left(A_{M}-A_{\mu}\right)^{1 / 2}\left((I-L) P_{1}+P_{2}-P_{1}\right)\left(A_{M}-A_{\mu}\right)^{1 / 2} .
\end{aligned}
$$

Hence, it follows from (2.3) that

$$
\begin{aligned}
& \operatorname{ran}\left(\widetilde{A}_{2}-\widetilde{A}_{1}\right)^{1 / 2}=\left(A_{M}-A_{\mu}\right)^{1 / 2}\left(\mathfrak{N} \ominus \mathfrak{N}_{2}\right), \\
& \operatorname{ran}\left(\widetilde{A}_{1}-A_{\mu}\right)^{1 / 2}=\left(A_{M}-A_{\mu}\right)^{1 / 2} L^{1 / 2} \mathfrak{N}_{1}, \\
& \operatorname{ran}\left(A_{M}-\widetilde{A}_{2}\right)^{1 / 2}=\left(A_{M}-A_{\mu}\right)^{1 / 2}\left\{(I-L)^{1 / 2} \mathfrak{N}_{1} \oplus\left(\mathfrak{N}_{2} \ominus \mathfrak{N}_{1}\right)\right\} .
\end{aligned}
$$

These equalities imply that

$$
\operatorname{ran}\left(\widetilde{A}_{2}-\widetilde{A}_{1}\right)^{1 / 2} \cap \operatorname{ran}\left(\widetilde{A}_{1}-A_{\mu}\right)^{1 / 2}=\{0\}, \operatorname{ran}\left(\widetilde{A}_{2}-\widetilde{A}_{1}\right)^{1 / 2} \cap \operatorname{ran}\left(A_{M}-\widetilde{A}_{2}\right)^{1 / 2}=\{0\} .
$$

According to Proposition 2.9 this proves the first two equalities in (4.1). Moreover, in view of $(4.3)$ one has $\operatorname{Ker}\left(\widetilde{A}_{2}-\widetilde{A}_{1}\right)=\operatorname{dom} A$, which completes the proof.

Remark 4.3. When $\left(A_{M}-A_{\mu}\right) \mathfrak{N} \neq \mathfrak{N}$ the proof of Proposition 4.2 shows that one can take $\mathfrak{N}_{1}=\{0\}$, in which case one obtains a pair $\left\{A_{\mu}, \widetilde{A}_{2}\right\}$ with $\widetilde{A}_{2} \neq A_{M}$. Then, according to Corollary 3.8 , one can also construct a pair $\left\{\widetilde{A}_{1}, A_{M}\right\}$ with $\widetilde{A}_{1} \neq A_{\mu}$. 
4.2. The case $\left(A_{M}-A_{\mu}\right) \mathfrak{N}=\mathfrak{N}$. Here the construction of a pair $\left\{\widetilde{A}_{1}, \widetilde{A}_{2}\right\}, \widetilde{A}_{1} \leq \widetilde{A}_{2}$, of $s c$-extensions of $A$, which satisfies the equalities in (4.1) is reduced to a problem of finding a nonnegative selfadjoint contraction in $\mathfrak{N}$ and a subspace $\mathfrak{M}$ of $\mathfrak{N}$ with the properties specified in the next lemma.

Lemma 4.4. Let $\left(A_{M}-A_{\mu}\right) \mathfrak{N}=\mathfrak{N}$. Then a pair $\left\{\widetilde{A}_{1}, \widetilde{A}_{2}\right\}, \widetilde{A}_{1} \leq \widetilde{A}_{2}$, of sc-extensions of $A$ satisfies the equalities (4.1) if and only if there exist a selfadjoint contraction $X_{1}$ in $\mathfrak{N}$ and a subspace $\mathfrak{M} \subset \mathfrak{N}$, such that

$$
\left\{\begin{array}{l}
0 \leq X_{1} \leq I, \operatorname{Ker}\left(I-X_{1}\right)=\{0\} \\
\operatorname{ran} X_{1}^{1 / 2} \cap \mathfrak{M}=\{0\} \\
\operatorname{ran}\left(I-X_{1}\right)^{1 / 2} \cap \mathfrak{M}^{\perp}=\{0\}
\end{array}\right.
$$

In this case the sc-extensions $\widetilde{A}_{1}$ and $\widetilde{A}_{2}$ satisfying the equalities (4.1) are given by

$$
\widetilde{A}_{j}=A_{\mu}+\left(A_{M}-A_{\mu}\right)^{1 / 2} X_{j}\left(A_{M}-A_{\mu}\right)^{1 / 2}, \quad j=1,2,
$$

where

$$
X_{2}=X_{1}+\left(I-X_{1}\right)^{1 / 2} P_{\mathfrak{M}}\left(I-X_{1}\right)^{1 / 2} .
$$

Proof. The sc-extensions $\widetilde{A}$ of $A$ are parametrized by (3.8) with $X \in\left[0, I_{\mathfrak{N}}\right]$. Hence, $\widetilde{A}_{1}$ and $\widetilde{A}_{2}, \widetilde{A}_{1} \leq \widetilde{A}_{2}$, can be presented in the form (4.6). Since $\left(A_{M}-A_{\mu}\right) \mathfrak{N}=\mathfrak{N}$, the equalities in (4.1) can be equivalently rewritten as

$$
\left\{\begin{array}{l}
0 \leq X_{1} \leq X_{2} \leq I \text { in } \mathfrak{N}, \\
\operatorname{Ker}\left(X_{2}-X_{1}\right)=\{0\}, \\
X_{1}:\left(X_{2}-X_{1}\right)=\left(I-X_{2}\right):\left(X_{2}-X_{1}\right)=0,
\end{array}\right.
$$

cf. (2.22) in Proposition 2.9. The condition $\operatorname{Ker}\left(X_{2}-X_{1}\right)=\{0\}$ implies that $\operatorname{Ker}\left(I-X_{1}\right)=$ $\{0\}$, since $0 \leq X_{1} \leq X_{2} \leq I$. By Lemma 2.8 the condition $\left(I-X_{2}\right):\left(X_{2}-X_{1}\right)=0$ is equivalent to

$$
X_{2}-X_{1}=\left(I-X_{1}\right)^{1 / 2} P\left(I-X_{1}\right)^{1 / 2},
$$

where $P$ is an orthogonal projection in $\mathfrak{N}$ onto $\mathfrak{M}:=\operatorname{ran} P$. By $(2.3), \operatorname{ran}\left(X_{2}-X_{1}\right)^{1 / 2}=$ $\left(I-X_{1}\right)^{1 / 2} \mathfrak{M}$ and hence the condition $X_{1}:\left(X_{2}-X_{1}\right)=0$ can be rewritten as

$$
\{0\}=\operatorname{ran} X_{1}^{1 / 2} \cap \operatorname{ran}\left(X_{2}-X_{1}\right)^{1 / 2}=\operatorname{ran} X_{1}^{1 / 2} \cap\left(I-X_{1}\right)^{1 / 2} \mathfrak{M} .
$$

Since $\operatorname{ker}\left(I-X_{1}\right)=\{0\}$, Corollary 2.6 shows that the condition (4.10) is equivalent to

$$
\operatorname{ran} X_{1}^{1 / 2} \cap \mathfrak{M}=\{0\}
$$

Moreover, (4.9) shows that $\operatorname{ker}\left(X_{2}-X_{1}\right)=\{0\}$ if and only if

$$
\operatorname{ran}\left(I-X_{1}\right)^{1 / 2} \cap \mathfrak{M}^{\perp}=\{0\},
$$

where $\mathfrak{M}^{\perp}=\mathfrak{N} \ominus \mathfrak{M}$. Therefore, $X_{1}$ and $\mathfrak{M}$ satisfy all the conditions in (4.5) and in view of (4.9) $X_{2}$ takes the form (4.7).

Now, assume that the conditions in (4.5) hold for some nonnegative selfadjoint contraction $X_{1}$ in $\mathfrak{N}$ and for some subspace $\mathfrak{M} \subset \mathfrak{N}$. Let $P_{\mathfrak{M}}$ be the orthogonal projection onto $\mathfrak{M}$ and let $X_{2}$ be defined by (4.7). Then clearly $0 \leq X_{1} \leq X_{2} \leq I$. The condition $\operatorname{ran}\left(I-X_{1}\right)^{1 / 2} \cap \mathfrak{M}^{\perp}=$ $\{0\}$ implies that $\operatorname{ker}\left(X_{2}-X_{1}\right)=\{0\}$. By construction $\left(I-X_{2}\right):\left(X_{2}-X_{1}\right)=0$, cf. Lemma 2.11. Moreover, the assumption $\operatorname{ran} X_{1}^{1 / 2} \cap \mathfrak{M}=\{0\}$ gives

$$
\operatorname{ran} X_{1}^{1 / 2} \cap \operatorname{ran}\left(X_{2}-X_{1}\right)^{1 / 2}=\operatorname{ran} X_{1}^{1 / 2} \cap\left(I-X_{1}\right)^{1 / 2} \mathfrak{M}=\{0\},
$$


cf. (4.10), (4.11), so that $X_{1}:\left(X_{2}-X_{1}\right)=0$. Thus, $X_{1}$ and $X_{2}$ satisfy all the conditions in (4.8). Consequently, the $s c$-extensions $\widetilde{A}_{1}$ and $\widetilde{A}_{2}$ defined by (4.6) satisfy the equalities in $(4.1)$.

Corollary 4.5. Let $\left(A_{M}-A_{\mu}\right) \mathfrak{N}=\mathfrak{N}$ and let $\left\{\widetilde{A}_{1}, \widetilde{A}_{2}\right\}, \widetilde{A}_{1} \leq \widetilde{A}_{2}$, be a pair of sc-extensions of $A$ satisfying (4.1). Then the equalities $\widetilde{A}_{1}=A_{\mu}$ and $\widetilde{A}_{2}=A_{M}$ are equivalent.

Proof. Assume that $\widetilde{A}_{1}=A_{\mu}$. Then $X_{1}=0$ and the third condition in (4.5) implies that $\mathfrak{M}=\mathfrak{N}$. It follows from (4.7) that $X_{2}=I$ and in view of (4.6) $\widetilde{A}_{2}=A_{M}$.

To obtain the converse statement, reverse the previous steps.

Remark 4.6. The existence of $X_{1}$ and $\mathfrak{M}$ in Lemma 4.4 is equivalent to the existence of a selfadjoint contraction $X_{2}$ in $\mathfrak{N}$ and a subspace $\mathfrak{L}$ of $\mathfrak{N}$ which satisfy the conditions

$$
\left\{\begin{array}{l}
0 \leq X_{2} \leq I, \text { Ker } X_{2}=\{0\}, \\
\operatorname{ran} X_{2}^{1 / 2} \cap \mathfrak{L}=\{0\}, \\
\operatorname{ran}\left(I-X_{2}\right)^{1 / 2} \cap \mathfrak{L}^{\perp}=\{0\} .
\end{array}\right.
$$

In this case, one can take $X_{1}=X_{2}^{1 / 2} P_{\mathfrak{L}} X_{2}^{1 / 2}$. The equivalence of the conditions in (4.13) and the conditions (4.5) in Lemma 4.4 can be easily checked. One can arrive at the conditions (4.13) when starting from the identity $X_{1}:\left(X_{2}-X_{1}\right)=0\left(\right.$ instead of $\left(I-X_{1}\right):\left(X_{2}-X_{1}\right)=0$ that was used in (4.9)).

Corollary 4.7. Let $\left(A_{M}-A_{\mu}\right) \mathfrak{N}=\mathfrak{N}$ and assume that the conditions (4.5) are satisfied by some subspace $\mathfrak{M}$ of $\mathfrak{N}$ and some selfadjoint contraction $X_{1}$ in $\mathfrak{N}$. If $\operatorname{dim} \mathfrak{M}<\infty$ or $\operatorname{dim} \mathfrak{M}^{\perp}<\infty$, then

$$
\widetilde{A}_{1}=A_{\mu} \quad \text { and } \quad \widetilde{A}_{2}=A_{M} .
$$

Proof. First, assume that $\operatorname{dim} \mathfrak{M}<\infty$. Then in view of (4.7) the operator $X_{2}-X_{1}$ is finite-dimensional and since $\operatorname{ker}\left(X_{2}-X_{1}\right)=\{0\}$, one concludes that $\operatorname{ran}\left(X_{2}-X_{1}\right)=\mathfrak{N}$. Consequently, $\operatorname{ran}\left(\widetilde{A}_{2}-\widetilde{A}_{1}\right)=\mathfrak{N}$ and hence the conditions (4.1) imply that $\widetilde{A}_{1}=A_{\mu}$ and $\widetilde{A}_{2}=A_{M}$.

Now, assume that $\operatorname{dim} \mathfrak{M}^{\perp}<\infty$. Then (4.7) implies that the operator

$$
I-X_{2}=\left(I-X_{1}\right)^{1 / 2}\left(I-P_{\mathfrak{M}}\right)\left(I-X_{1}\right)^{1 / 2}
$$

is finite-dimensional and $\operatorname{ran}\left(I-X_{2}\right)$ is closed. Since $\mathfrak{N}=\operatorname{ran} X_{2}^{1 / 2}+\operatorname{ran}\left(I-X_{2}\right)^{1 / 2}$, also ran $X_{2}^{1 / 2}$ must be closed. Moreover, the assumptions imply that $\operatorname{ker}\left(X_{2}-X_{1}\right)=\{0\}$, cf. (4.12). Then also $\operatorname{ker} X_{2}=0$ and since $\operatorname{ran} X_{2}$ is closed, $X_{2}$ is boundedly invertible. By Lemma 2.11 the property $X_{1}:\left(X_{2}-X_{1}\right)=0$ in (4.8) shows that

$$
X_{2}-X_{1}=X_{2}^{1 / 2} P X_{2}^{1 / 2}
$$

for some orthogonal projection $P$ in $\mathfrak{N}$. Hence, $\operatorname{ran}\left(X_{2}-X_{1}\right)$ is closed and the identity $\operatorname{ran}\left(X_{2}-X_{1}\right)=\mathfrak{N}$ follows from $\operatorname{ker}\left(X_{2}-X_{1}\right)=\{0\}$. Therefore, again from (4.1) one concludes that $\widetilde{A}_{1}=A_{\mu}$ and $\widetilde{A}_{2}=A_{M}$.

Corollary 4.7 shows that in the case where $\left(A_{M}-A_{\mu}\right) \mathfrak{N}=\mathfrak{N}$ for the existence of $\widetilde{A}_{1} \neq A_{\mu}$ and $\widetilde{A}_{2} \neq A_{M}$ it is necessary that the subspaces $\mathfrak{M}$ and $\mathfrak{M}^{\perp}=\mathfrak{N} \ominus \mathfrak{M}$ in Lemma 4.4 both are infinite-dimensional. Next it is shown how to construct an operator $X$ in $\mathfrak{N}$ and a subspace $\mathfrak{M}$ of $\mathfrak{N}$ which satisfy all the conditions in (4.5) and, such that $\operatorname{dim} \mathfrak{M}=\infty$ and $\operatorname{dim} \mathfrak{M}^{\perp}=\infty$. 
It is well known that there exist unbounded selfadjoint operators on infinite dimensional Hilbert spaces $\mathfrak{H}$, whose (dense) domains have a trivial intersection; see e.g. [21], [15] and the references therein. Consequently, there exist bounded nonnegative operators $F$ and $G$ in $\mathfrak{H}$, such that

$$
\overline{\operatorname{ran}} F=\overline{\operatorname{ran}} G=\mathfrak{H}, \quad \operatorname{ran} F \cap \operatorname{ran} G=\{0\} .
$$

Lemma 4.8. Let $\mathfrak{H}$ be an infinite-dimensional Hilbert space. Then there exist a contraction $X \neq 0$ in $\mathfrak{H}$ and a subspace $\mathfrak{M} \subset \mathfrak{H}$ which possess all the properties in (4.5).

Proof. Let $\mathfrak{K}$ be an infinite-dimensional subspace of $\mathfrak{H}$ with $\operatorname{dim} \mathfrak{K}=\operatorname{dim} \mathfrak{K}^{\perp}$. Then $\mathfrak{K}^{\perp}$ can be identified with $\mathfrak{K}$, and one can write $\mathfrak{H}$ as a direct sum $\mathfrak{H}=\mathfrak{K} \oplus \mathfrak{K}$. Let $F=F^{*} \geq 0$ and $G=G^{*}$ be bounded operators in the Hilbert space $\mathfrak{K}$ satisfying (4.14):

$$
\overline{\operatorname{ran}} F=\overline{\operatorname{ran}} G=\mathfrak{K}, \quad \operatorname{ran} F \cap \operatorname{ran} G=\{0\} .
$$

Without loss of generality one can assume that $\|F\|<1$. Then $F$ is contractive and $\operatorname{ker}(I-$ $\left.F^{2}\right)=\{0\}$. Now define

$$
X=\left(\begin{array}{cc}
F^{2} & 0 \\
0 & I-F^{2}
\end{array}\right), \quad \mathfrak{M}=\left\{\left(\begin{array}{c}
G h \\
h
\end{array}\right): h \in \mathfrak{K}\right\} .
$$

Then $X$ is a selfadjoint nonnegative contraction in $\mathfrak{H}$ with $\operatorname{ker} X=\{0\}$ and $\mathfrak{M}$ is a closed linear subspace of $\mathfrak{H}$. Assume that $v \in \operatorname{ran} X^{1 / 2} \cap \mathfrak{M}$. Then for some $h, x, y \in \mathfrak{K}$ one has

$$
v=\left(\begin{array}{c}
G h \\
h
\end{array}\right)=\left(\begin{array}{c}
F x \\
\left(I-F^{2}\right)^{1 / 2} y
\end{array}\right)
$$

Since $\operatorname{ran} F \cap \operatorname{ran} G=\{0\},(4.16)$ implies $F x=G h=0$. Due to $\operatorname{ker} F=\operatorname{ker} G=\{0\}$ one obtains $x=0, h=0$. Therefore, also $v=0$. This proves that $\operatorname{ran} X^{1 / 2} \cap \mathfrak{M}=\{0\}$.

Next observe that

$$
I-X=\left(\begin{array}{cc}
I-F^{2} & 0 \\
0 & F^{2}
\end{array}\right), \quad \mathfrak{M}^{\perp}=\left\{\left(\begin{array}{c}
k \\
-G k
\end{array}\right): k \in \mathfrak{K}\right\} .
$$

Clearly, $\operatorname{ker}(I-X)=\{0\}$. Furthermore, a similar argument as was used above shows that $\operatorname{ran}(I-X)^{1 / 2} \cap \mathfrak{M}^{\perp}=\{0\}$.

Observe, that the contraction $X \geq 0$ constructed in Lemma 4.8, in addition to (4.5), satisfies $\operatorname{ker} X=\{0\}$. As a consequence of Lemmas 4.4, 4.8 one obtains

Proposition 4.9. Let $\left(A_{M}-A_{\mu}\right) \mathfrak{N}=\mathfrak{N}$. Then there exists a pair $\left\{\widetilde{A}_{1}, \widetilde{A}_{2}\right\}$ of sc-extensions of $A$ which satisfies the equalities (4.1) but does not coincide with the pair $\left\{A_{\mu}, A_{M}\right\}$.

In the next theorem a characterization of all operators $B$ satisfying the conditions in (4.5) in Lemma 4.4 is given.

Theorem 4.10. Let $\mathfrak{N}$ be an infinite-dimensional Hilbert space, $\mathfrak{N}=\mathfrak{M}^{\perp} \oplus \mathfrak{M}$ be an orthogonal decomposition of $\mathfrak{N}$ such that $\mathfrak{M}$ and $\mathfrak{M}^{\perp}$ are infinite-dimensional subspaces. Then a bounded operator $B$ in $\mathfrak{N}$ satisfies the conditions

$$
\left\{\begin{array}{l}
0 \leq B \leq I, \\
\operatorname{ran} B^{1 / 2} \cap \mathfrak{M}=\{0\}, \\
\operatorname{ran}(I-B)^{1 / 2} \cap \mathfrak{M}^{\perp}=\{0\},
\end{array}\right.
$$

if and only if with respect to the decomposition $\mathfrak{N}=\mathfrak{M}^{\perp} \oplus \mathfrak{M} B=\left(B_{i j}\right)_{i, j=1}^{2}$ is of the form

$$
B=\left[\begin{array}{cc}
T^{*} D_{U^{*}}^{2} T & T^{*} U D_{U} \\
D_{U} U^{*} T & U^{*} U
\end{array}\right]
$$


where $U: \mathfrak{M} \rightarrow \mathfrak{M}^{\perp}$ is a strict contraction $(\|U f\|<\|f\|, f \in \mathfrak{M})$, and $T: \mathfrak{M}^{\perp} \rightarrow \mathfrak{M}^{\perp}$ is an isometry such that $\operatorname{ran} D_{U^{*}} \cap \operatorname{ker} T^{*}=\{0\}$.

Proof. Let $B$ be of the form (4.17) where $U$ and $T$ satisfy the given assumptions. Then with $h=x+y, x \in \mathfrak{M}^{\perp}, y \in \mathfrak{M}$ one obtains

$$
(B h, h)=\left\|D_{U^{*}} T x\right\|^{2}+2 \operatorname{Re}\left(U y, D_{U^{*}} T x\right)+\|U y\|^{2}=\left\|D_{U^{*}} T x+U y\right\|^{2} .
$$

Moreover, by isometry of $T$ one also has

$$
((I-B) h, h)=\left\|U^{*} T x\right\|^{2}-2 \operatorname{Re}\left(D_{U} y, U^{*} T x\right)+\left\|D_{U} y\right\|^{2}=\left\|U^{*} T x-D_{U} y\right\|^{2} .
$$

Hence, $0 \leq B \leq I$. Since $U$ is a strict contraction and $\operatorname{ran} D_{U^{*}} \cap \operatorname{ker} T^{*}=\{0\}$, one concludes that

Consequently,

$$
\overline{\operatorname{ran}} D_{U}=\mathfrak{M}, \overline{\operatorname{ran}} D_{U^{*}} T=\mathfrak{M}^{\perp} .
$$

$$
\inf \left\{(B(h-\varphi), h-\varphi): \varphi \in \mathfrak{M}^{\perp}\right\}=0, \inf \{((I-B)(h-\psi), h-\psi): \psi \in \mathfrak{M}\}=0 .
$$

According to (2.15) these relations mean that

$$
B_{\mathfrak{M}}=0, \quad(I-B)_{\mathfrak{M}^{\perp}}=0,
$$

or equivalently, that

$$
\operatorname{ran} B^{1 / 2} \cap \mathfrak{M}=\{0\}, \quad \operatorname{ran}(I-B)^{1 / 2} \cap \mathfrak{M}^{\perp}=\{0\} .
$$

To prove the converse, decompose $B=\left(B_{i j}\right)_{i, j=1}^{2}$ according to $\mathfrak{M}^{\perp} \oplus \mathfrak{M}$. By $(2.14)$ the condition $\operatorname{ran} B^{1 / 2} \cap \mathfrak{M}=\{0\}$ is equivalent to $B_{\mathfrak{M}}=0$. Hence, according to (2.26) $B_{22}=U^{*} U$, where $U=B_{11}^{(-1 / 2)} B_{12}: \mathfrak{M} \rightarrow \overline{\operatorname{ran}} B_{11}^{1 / 2}$ is a bounded operator. Now $I-B \geq 0$ can be rewritten as

$$
I-B=\left[\begin{array}{cc}
I-B_{11} & -B_{12} \\
-B_{12}^{*} & I-U^{*} U
\end{array}\right] \geq 0 .
$$

The condition $\operatorname{ran}(I-B)^{1 / 2} \cap \mathfrak{M}^{\perp}=\{0\}$ is equivalent to $(I-B)_{\mathfrak{M}^{\perp}}=0$, and hence in view of (2.26) $I-B_{11}=Z^{*} Z$, where $Z=D_{U}^{-1} B_{12}^{*}: \mathfrak{M}^{\perp} \rightarrow \overline{\operatorname{ran}} D_{U}$ is a bounded operator. Hence, $B_{11}^{1 / 2}=D_{Z}$ and

$$
B_{12}^{*}=Z^{*} D_{U}=D_{Z} U
$$

It follows from

$$
0 \leq B=\left[\begin{array}{cc}
D_{Z}^{2} & D_{Z} U \\
U^{*} D_{Z} & U^{*} U
\end{array}\right]=\left[\begin{array}{c}
D_{Z} \\
U^{*}
\end{array}\right]\left[\begin{array}{ll}
D_{Z} & U
\end{array}\right] \leq I
$$

that $0 \leq D_{Z}^{2}+U U^{*} \leq I_{\mathfrak{M}^{\perp}}$, so that $D_{Z}^{2} \leq D_{U^{*}}^{2}$. Hence, there is a contraction $T: \mathfrak{M}^{\perp} \rightarrow$ $\overline{\operatorname{ran}} D_{U^{*}}$, such that

$$
D_{Z}=D_{U^{*}} T \text {. }
$$

Here $\operatorname{ran} D_{Z} \subset \operatorname{ran} D_{U^{*}}$ and $\operatorname{ker} D_{Z} \supseteq \operatorname{ker} D_{U^{*}}$ and one can write $D_{Z}=T^{*} D_{U^{*}}$ where $T^{*}: \overline{\operatorname{ran}} D_{U^{*}} \rightarrow \mathfrak{M}^{\perp}$ is the adjoint of $T$. The identities (4.18) and (4.19) imply

$$
D_{U} Z=U^{*} D_{Z}=U^{*} D_{U^{*}} T=D_{U} U^{*} T \text {. }
$$

Since $U^{*} \overline{\operatorname{ran}} D_{U^{*}} \subseteq \overline{\operatorname{ran}} D_{U}$, (4.20) gives

$$
Z=U^{*} T \text {. }
$$

Besides,

$$
D_{Z}^{2}=T^{*} D_{U^{*}}^{2} T=T^{*} T-T^{*} U U^{*} T
$$


and on the other hand

$$
D_{Z}^{2}=I_{\mathfrak{M}^{\perp}}-Z^{*} Z=I_{\mathfrak{M}^{\perp}}-T^{*} U U^{*} T .
$$

Thus, $T: \mathfrak{M}^{\perp} \rightarrow \overline{\operatorname{ran}} D_{U^{*}}$ is an isometry. If $D_{Z} x=0$ then

$$
\|x\|^{2}=\left\|U^{*} T x\right\|^{2} \leq\|T x\|^{2}=\|x\|^{2} .
$$

It follows that $\left\|U^{*} T x\right\|=\|T x\|=\|x\|$. Since $\operatorname{ran} T \subseteq \overline{\operatorname{ran}} D_{U^{*}}$, one has $\|T x\|=0$ and hence $x=0$. This shows that $\operatorname{Ker} D_{Z}=0$. From ker $D_{Z} \supset \operatorname{ker} D_{U^{*}}$ one obtains $\operatorname{ker} D_{U^{*}}=0$, so that $U^{*}$, and therefore also $U$, is a strict contraction. Moreover, from (4.19) it follows that

$$
\operatorname{ran} D_{U^{*}} \cap \operatorname{ker} T^{*}=\{0\} .
$$

Therefore, $B$ takes the form (4.17) where $U$ and $T$ satisfy the given conditions.

Observe that when the operator $B$ is of the form (4.17) with the corresponding parameters $U$ and $T$ satisfying the conditions of Theorem 4.10 then

$$
\operatorname{ker} B=\{0\} \Longleftrightarrow\left\{\begin{array}{l}
\operatorname{ker} U=\{0\} \\
\operatorname{ran} U \cap \operatorname{ran}\left(D_{U^{*}} T\right)=\{0\}
\end{array}\right.
$$

Since $T$ is an isometry, Corollary 2.5 gives

$$
\operatorname{ran} U \cap \operatorname{ran}\left(D_{U^{*}} T\right)=\{0\} \Longleftrightarrow \operatorname{ran} U \cap \operatorname{ran} T=\{0\} .
$$

Thus the following assertion is proved.

Corollary 4.11. Let $\mathfrak{N}$ be an infinite-dimensional Hilbert space, $\mathfrak{N}=\mathfrak{M}^{\perp} \oplus \mathfrak{M}$ be an orthogonal decomposition of $\mathfrak{N}$ such that $\mathfrak{M}$ and $\mathfrak{M}^{\perp}$ are infinite-dimensional subspaces. Then a bounded operator $B$ in $\mathfrak{N}$ satisfies the conditions

$$
\left\{\begin{array}{l}
0 \leq B \leq I \\
\operatorname{ker} B=\{0\} \\
\operatorname{ran} B^{1 / 2} \cap \mathfrak{M}=\{0\} \\
\operatorname{ran}(I-B)^{1 / 2} \cap \mathfrak{M}^{\perp}=\{0\}
\end{array}\right.
$$

if and only if with respect to the decomposition $\mathfrak{N}=\mathfrak{M}^{\perp} \oplus \mathfrak{M} B=\left(B_{i j}\right)_{i, j=1}^{2}$ is of the form

$$
B=\left[\begin{array}{cc}
T^{*} D_{U^{*}}^{2} T & T^{*} U D_{U} \\
D_{U} U^{*} T & U^{*} U
\end{array}\right]
$$

where $U: \mathfrak{M} \rightarrow \mathfrak{M}^{\perp}$ is a strict contraction $(\|U f\|<\|f\|, f \in \mathfrak{M})$ and $T: \mathfrak{M}^{\perp} \rightarrow \mathfrak{M}^{\perp}$ is an isometry, such that

$$
\left\{\begin{array}{l}
\operatorname{ker} U=\{0\}, \\
\operatorname{ran} U \cap \operatorname{ran} T=\{0\}, \\
\operatorname{ran} D_{U^{*}} \cap \operatorname{ker} T^{*}=\{0\} .
\end{array}\right.
$$

In order to construct a strict contraction $U$ and an isometry $T$ satisfying (4.21) it is necessary and sufficient to construct a contraction $U: \mathfrak{M} \rightarrow \mathfrak{M}^{\perp}$ and a subspace $\mathfrak{K} \subset \mathfrak{M}^{\perp}$ such that

$$
\left\{\begin{array}{l}
\operatorname{ker} U=\{0\}, \\
\operatorname{ker} D_{U^{*}}=\{0\}, \\
\operatorname{ran} U \cap \mathfrak{K}=\{0\}, \\
\operatorname{ran} D_{U^{*}} \cap\left(\mathfrak{M}^{\perp} \ominus \mathfrak{K}\right)=\{0\} .
\end{array}\right.
$$


Clearly, the subspaces $\mathfrak{K}$ and $\mathfrak{M}^{\perp} \ominus \mathfrak{K}$ satisfying the above conditions should be infinitedimensional. By Lemma 4.8 there exist a nonnegative selfadjoint contraction $X$ in $\mathfrak{M}^{\perp}$ and a subspace $\mathfrak{K} \subset \mathfrak{M}^{\perp}$ such that

$$
\operatorname{ker} X=\operatorname{ker}(I-X)=\{0\}, \operatorname{ran} X^{1 / 2} \cap \mathfrak{K}=\{0\}, \operatorname{ran}(I-X)^{1 / 2} \cap\left(\mathfrak{M}^{\perp} \ominus \mathfrak{K}\right)=\{0\} .
$$

Let $W$ be a unitary operator from $\mathfrak{M}$ onto $\mathfrak{M}^{\perp}$ and let $U=X^{1 / 2} W$. Then $U: \mathfrak{M} \rightarrow \mathfrak{M}^{\perp}$ is a contraction, $\operatorname{ker} U=\{0\}, \operatorname{ran} U \cap \mathfrak{K}=\{0\}, D_{U^{*}}=(I-X)^{1 / 2}$, $\operatorname{ker} D_{U^{*}}=\{0\}$ and $\operatorname{ran} D_{U^{*}} \cap\left(\mathfrak{M}^{\perp} \ominus \mathfrak{K}\right)=\{0\}$.

Remark 4.12. In [33] K. Schmüdgen has solved a closely related problem: for a closed unbounded operator $B$ in a Hilbert space $\mathfrak{H}$ construct an orthogonal projection $P$ in $\mathfrak{H}$, such that $P \mathfrak{H} \cap \operatorname{dom} B=(I-P) \mathfrak{H} \cap \operatorname{dom} B=\{0\}$.

Another closely related question has been treated by J.E. Brasche, H. Neidhardt in [13].

Remark 4.13. The main consequences of Subsections 4.1 and 4.2 are collected in the Theorem which was stated already in the Introduction.

4.3. Some analytic descriptions. The results in the previous two subsections can now be interpreted in analytical terms via the notion of $\widetilde{Q}_{1}$ - and $\widetilde{Q}_{2}$-functions. For this purpose the following subclasses of $\mathbf{N}_{\mathfrak{N}}[-1,1]$, where $\mathfrak{N}$ is a Hilbert space, are introduced.

Definition 4.14. A function $\widetilde{Q}_{1}(z)$ is said to belong to the subclass $\mathfrak{S}_{\mu}(\mathfrak{N})$ of $\mathbf{N}_{\mathfrak{N}}[-1,1]$ if: 1) $s-\lim _{z \rightarrow \infty} \widetilde{Q}_{1}(z)=I$,

2) $\lim _{z \uparrow-1}\left(\widetilde{Q}_{1}(z) h, h\right)=+\infty$, for all $h \in \mathfrak{N} \backslash\{0\}$,

3) $s-\lim _{z \downarrow 1} \widetilde{Q}_{1}(z)=0$.

Definition 4.15. A function $\widetilde{Q}_{2}(z)$ is said to belong to the subclass $\mathfrak{S}_{M}(\mathfrak{N})$ of $\mathbf{N}_{\mathfrak{N}}[-1,1]$ if: 1) $s-\lim _{z \rightarrow \infty} \widetilde{Q}_{2}(z)=-I$

2) $\lim _{z \downarrow 1}\left(\widetilde{Q}_{2}(z) h, h\right)=-\infty$, for all $h \in \mathfrak{N} \backslash\{0\}$,

3) $s-\lim _{z \uparrow-1} \widetilde{Q}_{2}(z)=0$.

It follows from (3.18) and (3.22) that the function $Q_{\mu}$ associated with the extension $A_{\mu}$ of $A$ via (3.14) belongs to the subclass $\mathfrak{S}_{\mu}(\mathfrak{N})$ and similarly the function $Q_{M}$ associated to the extension $A_{M}$ of $A$ via (3.15) belongs to the subclass $\mathfrak{S}_{M}(\mathfrak{N})$. Observe, that by Theorem 3.2 the limit conditions 1) and 3) in the definition of the classes $\mathfrak{S}_{\mu}(\mathfrak{N})$ and $\mathfrak{S}_{M}(\mathfrak{N})$ guarantee that the functions $-\widetilde{Q}_{1}^{-1}(z)$ and $-\widetilde{Q}_{2}^{-1}(z)$ belong to the class $\mathbf{N}_{\mathcal{H}}[-1,1]$. The main consequences of the above results for the classes $\mathfrak{S}_{\mu}(\mathfrak{N})$ and $\mathfrak{S}_{M}(\mathfrak{N})$ are given in the next theorem.

Theorem 4.16. Let $\widetilde{A}_{1}, \widetilde{A}_{2} \in\left[A_{\mu}, A_{M}\right], \widetilde{A}_{1} \leq \widetilde{A}_{2}$, be sc-extensions of $A$ and let (3.13) be satisfied. Define in $\mathfrak{N}=\mathfrak{H} \ominus \operatorname{dom} A$ the functions $\widetilde{Q}_{1}$ and $\widetilde{Q}_{2}$ by (3.14) and (3.15), respectively. Then the following statements are equivalent:

(i) $\widetilde{Q}_{1}$ belongs to $\mathfrak{S}_{\mu}(\mathfrak{N})$;

(ii) $\widetilde{Q}_{2}\left(=-\widetilde{Q}_{1}^{-1}\right)$ belongs to $\mathfrak{S}_{M}(\mathfrak{N})$;

(iii) the conditions in (4.1) are satisfied. 
If one of these conditions holds and $\operatorname{dim} \mathfrak{N}<\infty$ then $\widetilde{Q}_{1}(z)$ is the $Q_{\mu}$-function of $A$ and $\widetilde{Q}_{2}(z)$ is the $Q_{M}$-function of $A$. However, if $\operatorname{dim} \mathfrak{N}=\infty$ then there are pairs $\left\{\widetilde{A}_{1}, \widetilde{A}_{2}\right\} \neq\left\{A_{\mu}, A_{M}\right\}$, such that $\widetilde{Q}_{1}\left(\neq Q_{\mu}\right)$ belongs to $\mathfrak{S}_{\mu}(\mathfrak{N})$ and $\widetilde{Q}_{2}\left(\neq Q_{M}\right)$ belongs to $\mathfrak{S}_{M}(\mathfrak{N})$.

Proof. Clearly, the function $\widetilde{Q}_{j}, j=1,2$, defined by (3.14), (3.15) belongs to $\mathbf{N}_{\mathfrak{N}}[-1,1]$ and in view of (3.18) it has the property 1$)$ in the definition of the class $\mathfrak{S}_{j}(\mathfrak{N})$. According to Lemma 3.7 the conditions 2) and 3) in the definition of $\mathfrak{S}_{j}(\mathfrak{N})$ are, in each case $j=1,2$, equivalent to the conditions in (4.1). This proves the equivalence of (i)-(iii).

The second part of the theorem follows now from Corollary 3.9 and Propositions 4.2, 4.9.

Theorem 4.16 means that the characterizations of $Q_{\mu^{-}}$and $Q_{M^{-}}$functions of M.G. Krein and I.E. Ovcharenko in [26] are not valid in case $\operatorname{dim} \mathfrak{N}=\infty$. Of course, in view of Lemma 3.7 it is possible to state also more specific results for the functions $\widetilde{Q}_{1}$ in (3.14) and $\widetilde{Q}_{2}$ in (3.15) separately.

\section{THE INVERSE PROBLEM}

Let $\mathfrak{N}$ be a Hilbert space. In this section the following inverse problem is considered: If a function $\widetilde{Q}_{1}$ belongs to the subclass $\mathfrak{S}_{\mu}(\mathfrak{N})$, can one represent it in the form (3.14) or: if a function $\widetilde{Q}_{2}$ belongs to the subclass $\mathfrak{S}_{M}(\mathfrak{N})$, can one represent it in the form (3.15). More precisely, the following result is true.

Theorem 5.1. Assume that $\widetilde{Q}_{1} \in \mathfrak{S}_{\mu}(\mathfrak{N})$. Then there exist a Hilbert space $\mathfrak{H} \supset \mathfrak{N}$, a Hermitian operator $A$ in $\mathfrak{H}$ defined on $\operatorname{dom} A=\mathfrak{H} \ominus \mathfrak{N}$, and sc-extensions $\widetilde{A}_{1}$ and $\widetilde{A}_{2}$ of $A$, such that

$$
\begin{aligned}
& \widetilde{A}_{1} \leq \widetilde{A}_{2}, \operatorname{Ker}\left(\widetilde{A}_{2}-\widetilde{A}_{1}\right)=\operatorname{dom} A \\
& \left(\widetilde{A}_{1}-A_{\mu}\right):\left(\widetilde{A}_{2}-\widetilde{A}_{1}\right)=\left(A_{M}-\widetilde{A}_{2}\right):\left(\widetilde{A}_{2}-\widetilde{A}_{1}\right)=0
\end{aligned}
$$

and

$$
\begin{aligned}
\widetilde{Q}_{1}(z) & =\left[\left(\widetilde{A}_{2}-\widetilde{A}_{1}\right)^{1 / 2}\left(\widetilde{A}_{1}-z I\right)^{-1}\left(\widetilde{A}_{2}-\widetilde{A}_{1}\right)^{1 / 2}+I\right] \mid \mathfrak{N}, \\
-\widetilde{Q}_{1}^{-1}(z) & =\left[\left(\widetilde{A}_{2}-\widetilde{A}_{1}\right)^{1 / 2}\left(\widetilde{A}_{2}-z I\right)^{-1}\left(\widetilde{A}_{2}-\widetilde{A}_{1}\right)^{1 / 2}-I\right] \mid \mathfrak{N} .
\end{aligned}
$$

Proof. It follows from Theorem 3.1 that there a nondecreasing operator function $F(t)$ with values in $\mathcal{L}(\mathfrak{N})$ and normalized by $F(-1)=0$, such that

$$
\widetilde{Q}_{1}(z)=I+\int_{-1}^{1} \frac{d F(t)}{t-z}
$$

where the identity $\widetilde{Q}_{1}(\infty)=I$ has been used. Let $\widetilde{C}=F(1)$. Then (5.2) shows that

$$
\widetilde{C}=\lim _{z \rightarrow \infty} z\left(I-\widetilde{Q}_{1}(z)\right)
$$

Since $0 \leq F(t) \leq \widetilde{C}$, one can write $F(t)=\widetilde{C}^{1 / 2} S(t) \widetilde{C}^{1 / 2}$, for a nondecreasing function $S(t)$ satisfying $S(-1)=0, S(1)=I$. According to M.A. Naumark's theorem [14] there exist a Hilbert space $\mathfrak{H} \supset \mathfrak{N}$ and an orthogonal spectral function $E(t)$, such that $S(t)=P_{\mathfrak{N}} E(t)\lceil\mathfrak{N}$ where $P_{\mathfrak{N}}$ is the orthogonal projection in $\mathfrak{H}$ onto $\mathfrak{N}$. Define the selfadjoint contraction $\widetilde{A}_{1}$ by

$$
\widetilde{A}_{1}=\int_{-1}^{1} t d E(t)
$$


and its restriction to $\operatorname{dom} A=\mathfrak{H} \ominus \mathfrak{N}$ by $A=\widetilde{A}_{1}\lceil\operatorname{dom} A$. Then $A$ is a Hermitian contraction and $\widetilde{A}_{1}$ is an $s c$-extension of $A$. Replacing $\widetilde{C}$ by $\widetilde{C} P_{\mathfrak{N}}$ one may interpret $\widetilde{C}$ as a mapping in $\mathfrak{H}$. Then one can write

$$
\widetilde{Q}_{1}(z)=I+\widetilde{C}^{1 / 2}\left(\widetilde{A}_{1}-z I\right)^{-1} \widetilde{C}^{1 / 2}
$$

Now, let $A_{\mu}$ and $A_{M}$ be the extremal sc-extensions of $A$ determining the corresponding operator interval. The condition 2) in the definition of the class $\mathfrak{S}_{\mu}(\mathfrak{N})$ shows that $\operatorname{ker} \widetilde{C}=$ $\operatorname{dom} A$. The condition 3) and the formula (2.2) imply that $\operatorname{ran} \widetilde{C}^{1 / 2} \subseteq \operatorname{ran}\left(I-\widetilde{A}_{1}\right)^{1 / 2}$ and, moreover, that $\left(I-\widetilde{A}_{1}\right)^{(-1 / 2)} C^{1 / 2}$ is a partial isometry with the initial space $\mathfrak{N}$. From Lemma 2.11 one concludes that

$$
\widetilde{C}:\left(I-\widetilde{A}_{1}-\widetilde{C}\right)=0 .
$$

Using (2.30) and (3.9) one obtains

$$
\widetilde{C}_{\mathfrak{N}}:\left(I-\widetilde{A}_{1}-\widetilde{C}\right)=\widetilde{C}:\left(I-\widetilde{A}_{1}-\widetilde{C}\right)_{\mathfrak{N}}=\widetilde{C}:\left(\left(I-\widetilde{A}_{1}\right)_{\mathfrak{N}}-\widetilde{C}\right)=\widetilde{C}:\left(A_{M}-\widetilde{A}_{1}-\widetilde{C}\right),
$$

so that the condition (5.4) is equivalent to $\widetilde{C}:\left(A_{M}-\widetilde{A}_{1}-\widetilde{C}\right)=0$. Thus, by Lemma 2.11

$$
\widetilde{C}=\left(A_{M}-\widetilde{A}_{1}\right)^{1 / 2} \widetilde{P}\left(A_{M}-\widetilde{A}_{1}\right)^{1 / 2}
$$

for some orthogonal projection $\widetilde{P}$ with ran $\widetilde{P} \subseteq \mathfrak{N}$. Now define $\widetilde{A}_{2}$ by

$$
\widetilde{A}_{2}=\widetilde{A}_{1}+\widetilde{C} \text {. }
$$

Then

$$
A_{M}-\widetilde{A}_{2}=\left(A_{M}-A_{1}\right)^{1 / 2}(I-\widetilde{P})\left(A_{M}-\widetilde{A}_{1}\right)^{1 / 2} .
$$

Therefore, $\widetilde{A}_{2} \in\left[A_{\mu}, A_{M}\right]$ which means that $\widetilde{A}_{2}$ is an $s c$-extension of $A$. Moreover,

$$
\operatorname{ran}\left(A_{M}-\widetilde{A}_{2}\right)^{1 / 2} \cap \operatorname{ran}\left(\widetilde{A}_{2}-\widetilde{A}_{1}\right)^{1 / 2}=\{0\},
$$

or equivalently, $\left(\widetilde{A}_{2}-\widetilde{A}_{1}\right):\left(A_{M}-\widetilde{A}_{2}\right)=0$. It follows from the condition 2$)$ in the definition of the class $\mathfrak{S}_{\mu}(\mathfrak{N})$ and $(2.2)$ that $\operatorname{ker} \widetilde{C}=\operatorname{dom} A$ and

$$
\operatorname{ran}\left(I+\widetilde{A}_{1}\right)^{1 / 2} \cap \operatorname{ran}\left(\widetilde{A}_{2}-\widetilde{A}_{1}\right)^{1 / 2}=\{0\} .
$$

Therefore, $\left(I+\widetilde{A}_{1}\right):\left(\widetilde{A}_{2}-\widetilde{A}_{1}\right)=0$ and by Remark 4.1 also $\left(\widetilde{A}_{1}-A_{\mu}\right):\left(\widetilde{A}_{2}-\widetilde{A}_{1}\right)=0$.

Remark 5.2. In the proof for [26, Theorem 2.2] M.G. Kre ̌n and I.E. Ovcharenko constructed the operator representation (5.3) for $\widetilde{Q}_{1}(z)$ : for the convenience of reader their arguments are repeated in the first part of the above proof. However, their statement that $\widetilde{C}$ is equal to $\left(I-\widetilde{A}_{1}\right)_{\mathfrak{N}}=A_{M}-\widetilde{A}_{1}$ does not hold in general when $\operatorname{dim} \mathfrak{N}=\infty$. This becomes clear from the proof given above, since in general $\operatorname{ran} \widetilde{P} \neq \mathfrak{N}$ in (5.5). It is also clear from (5.5) that if $\operatorname{dim} \mathfrak{N}<\infty$ then $\operatorname{ran} \widetilde{P}=\mathfrak{N}$ due to ker $\widetilde{C}=\operatorname{dom} A$.

Corollary 5.3. Let $\operatorname{dim} \mathfrak{N}<\infty$ and $\widetilde{Q}_{1}(z) \in \mathfrak{S}_{\mu}(\mathfrak{N})$. Then $\widetilde{Q}_{1}(z)$ and $\widetilde{Q}_{2}(z)=-\widetilde{Q}_{1}^{-1}(z)$ are the $Q_{\mu}$ - and $Q_{M}$-functions of some Hermitian contraction.

Recall from [26] that two Hermitian operators $A$ and $A^{\prime}$ defined on the subspaces $\operatorname{dom} A$ and $\operatorname{dom} A^{\prime}$ of the Hilbert spaces $\mathfrak{H}=\operatorname{dom} A \oplus \mathfrak{N}$ and $\mathfrak{H}^{\prime}=\operatorname{dom} A^{\prime} \oplus \mathfrak{N}$, respectively, are said to be unitarily equivalent, if there is a unitary operator $U$ from $\mathfrak{H}$ onto $\mathfrak{H}^{\prime}$, such that

$$
U \uparrow \mathfrak{N}=\mathfrak{N}, \quad U(\operatorname{dom} A)=\operatorname{dom} A^{\prime}, \quad U A=A^{\prime} U .
$$

Moreover, $A$ in $\mathfrak{H}$ is said to be simple if there is no nontrivial subspace of $\mathfrak{H}$ invariant under A. An equivalent condition due to M.G. Krĕn and I.E. Ovcharenko [26, Lemma 2.1] is 
that the subspace $\mathfrak{N}=\mathfrak{H} \ominus \operatorname{dom} A$ is generating for some (equivalently for every) selfadjoint extension $\widetilde{A}$ of $A$ :

$$
\mathfrak{H}=\overline{\operatorname{span}}\left\{\widetilde{A}^{n} \mathfrak{N}: n=0,1, \ldots\right\} .
$$

In [26] it is shown that the simple part of the Hermitian contraction $A$ is uniquely determined by its $Q_{\mu}$-function up to unitary equivalence. An analogous statement holds for functions belonging to the classes $\mathfrak{S}_{\mu}(\mathfrak{N})$ and $\mathfrak{S}_{M}(\mathfrak{N})$. Moreover, the following generalization of this result for the pair $\left\{\widetilde{A}_{1}, \widetilde{A}_{2}\right\}$ of $s c$-extensions of $A$ is also true.

Proposition 5.4. Let $A$ and $A^{\prime}$ be simple Hermitian contractions in $\mathfrak{H}=\operatorname{dom} A \oplus \mathfrak{N}$ and $\mathfrak{H}^{\prime}=\operatorname{dom} A^{\prime} \oplus \mathfrak{N}$, respectively, and let $\widetilde{Q}_{1}(z)$ and $\widetilde{Q}_{1}^{\prime}(z)$ be defined via $(3.14)\left(\widetilde{Q}_{2}(z)\right.$ and $\widetilde{Q}_{2}^{\prime}(z)$ be defined via (3.15)) with the pair $\left\{\widetilde{A}_{1}, \widetilde{A}_{2}\right\}$ and $\left\{\widetilde{A}_{1}^{\prime}, \widetilde{A}_{2}^{\prime}\right\}$, respectively. If $\widetilde{Q}_{1}(z)$ and $\widetilde{Q}_{1}^{\prime}(z)$ are equal, then $A$ and $A^{\prime}$ and the pairs $\left\{\widetilde{A}_{1}, \widetilde{A}_{2}\right\}$ and $\left\{\widetilde{A}_{1}^{\prime}, \widetilde{A}_{2}^{\prime}\right\}$ are unitary equivalent with the same unitary operator $U$.

Proof. Since $\widetilde{Q}_{2}(z)=-\widetilde{Q}_{1}(z)^{-1}$, it is enough to prove the assertion for the function $\widetilde{Q}_{1}(z)$. The unitary operator $U$ providing the unitary equivalence of $A, A^{\prime}$ and $\widetilde{A}_{1}, \widetilde{A}_{1}^{\prime}$ can be constructed in the usual manner, for instance, via obvious modifications of the arguments given in [26, Theorem 2.2]. Next observe that $\lim _{z \rightarrow \infty} z\left(I-\widetilde{Q}_{1}(z)\right)=\widetilde{A}_{2}-\widetilde{A}_{1}$ in view of (3.14). Now, from the equality $\widetilde{Q}_{1}(z)=\widetilde{Q}_{1}^{\prime}(z)$ one obtains the unitary equivalence of $\widetilde{A}_{2}$ and $\widetilde{A}_{2}^{\prime}$ with the same operator $U$.

\section{Characterization of $Q_{\mu}$ - And $Q_{M}$-FunCtions}

In this section the following problem is considered: under what conditions a function $Q_{1}$ which belongs to $\mathfrak{S}_{\mu}(\mathfrak{N})\left(Q_{2} \in \mathfrak{S}_{M}(\mathfrak{N})\right)$ is a $Q_{\mu}$-function $\left(Q_{M}\right.$-function, respectively) as defined in [26]. In other words, what additional conditions are needed in order to find a Hilbert space $\mathfrak{H} \supset \mathfrak{N}$ and a Hermitian contraction $A$ in $\mathfrak{H}$, such that $Q_{1}\left(Q_{2}\right)$ takes the form in $(3.16)$ :

$$
\begin{aligned}
& Q_{\mu}(z)=\left[\left(A_{M}-A_{\mu}\right)^{1 / 2}\left(A_{\mu}-z I\right)^{-1}\left(A_{M}-A_{\mu}\right)^{1 / 2}+I\right]\lceil\mathfrak{N}, \\
& Q_{M}(z)=\left[\left(A_{M}-A_{\mu}\right)^{1 / 2}\left(A_{M}-z I\right)^{-1}\left(A_{M}-A_{\mu}\right)^{1 / 2}-I\right]\lceil\mathfrak{N} .
\end{aligned}
$$

In view of Theorem 4.16 the limit conditions defining the classes $\mathfrak{S}_{\mu}(\mathfrak{N})$ and $\mathfrak{S}_{M}(\mathfrak{N})$ are not enough to characterize $Q_{\mu^{-}}$and $Q_{M^{-}}$-functions.

Let the function $V(z)$ belong to $\mathbf{N}_{\mathfrak{N}}[-1,1]$. Theorem 5.1 implies that $V(z)$ has an operator representation of the form

$$
V(z)=V(\infty)+G(B-z I)^{-1} G,
$$

where $B$ is a selfadjoint contraction in some Hilbert space $\mathfrak{H} \supset \mathfrak{N}$ and $G \geq 0$ is a bounded nonnegative selfadjoint operator in $\mathfrak{N}$ satisfying

$$
G^{2}=\lim _{z \rightarrow \infty} z(V(\infty)-V(z)) .
$$

Suppose that $\operatorname{Ker} G=\{0\}$ and let $\operatorname{ran} G$ be equipped with the inner product

$$
(f, g)_{+}=\left(G^{-1} f, G^{-1} g\right) .
$$

Then $\operatorname{ran} G$ becomes a Hilbert space which is denoted by $\mathfrak{N}_{+}^{V}$. Let $\mathfrak{N}_{+}^{V} \subseteq \mathfrak{N} \subseteq \mathfrak{N}_{-}^{V}$ be the corresponding rigged Hilbert space, cf. [12]. The operator $G$ is an isometry which maps $\mathfrak{N}$ onto $\mathfrak{N}_{+}^{V}$. Let $G^{\times} \in \mathcal{L}\left(\mathfrak{N}_{-}^{V}, \mathfrak{N}\right)$ be the adjoint operator. Since $G$ is a selfadjoint operator in 
$\mathfrak{N}$, the operator $G^{\times}$is the continuation of $G$. Moreover, $G^{\times}$is an isometry from $\mathfrak{N}_{-}^{V}$ onto $\mathfrak{N}$. As a consequence, for each $z \in \operatorname{Ext}[-1,1]$ the function

$$
U(z)=V(z)-V(\infty)=G(B-z I)^{-1} G
$$

has the continuation $G(B-z I)^{-1} G^{\times}$with values in $\mathcal{L}\left(\mathfrak{N}_{-}^{V}, \mathfrak{N}_{+}^{V}\right)$. The definition of classes $\mathfrak{S}_{\mu}(\mathfrak{N})$ and $\mathfrak{S}_{\mu}(\mathfrak{N})$, yields the following result.

Proposition 6.1. Let $\widetilde{Q}_{1}(z) \in \mathfrak{S}_{\mu}(\mathfrak{N})$. Then the triplet $\mathfrak{N}_{+}^{\widetilde{Q}_{1}} \subseteq \mathfrak{N} \subseteq \mathfrak{N}_{-}^{\widetilde{Q}_{1}}$ coincides with the triplet $\mathfrak{N}_{+}^{-\widetilde{Q}_{1}^{-1}} \subseteq \mathfrak{N} \subseteq \mathfrak{N}_{-}^{-\widetilde{Q}_{1}^{-1}}$.

Proof. By Theorem 5.1 $\widetilde{Q}_{1}(z)$ and $-\widetilde{Q}_{1}^{-1}(z)$ have operator representations of the form (5.1). From $(5.1)$ it is clear that $\lim _{z \rightarrow \infty} z(V(\infty)-V(z))=\left(\widetilde{A}_{2}-\widetilde{A}_{1}\right)\left\lceil\mathfrak{N}\right.$ in both cases $V(z)=\widetilde{Q}_{1}(z)$ and $V(z)=-\widetilde{Q}_{1}^{-1}(z)$.

Theorem 6.2. Let $\mathfrak{N}$ be a Hilbert space and let $\widetilde{Q}_{1}(z)$ belong to $\mathfrak{S}_{\mu}(\mathfrak{N})$. Then $\widetilde{Q}_{1}(z)$ is a $Q_{\mu}$-function of some Hermitian contraction if and only if the following conditions are fulfilled:

$$
\lim _{x \uparrow-1}\left(\left(\widetilde{Q}_{1}(x)-I\right) f, f\right)=\lim _{x \downarrow 1}\left(\left(\widetilde{Q}_{1}^{-1}(x)-I\right) f, f\right)=+\infty \quad \text { for all } \quad f \in \mathfrak{N}_{-}^{\widetilde{Q}_{1}} \backslash\{0\} .
$$

Proof. By Theorem 5.1 there exist a Hilbert space $\mathfrak{H} \supset \mathfrak{N}$, a Hermitian contraction $A$ in $\mathfrak{H}$ defined on $\operatorname{dom} A=\mathfrak{H} \ominus \mathfrak{N}$, and $s c$-extensions $\widetilde{A}_{1}, \widetilde{A}_{2}$ of $A$, such that

$$
\begin{aligned}
& \widetilde{A}_{1} \leq \widetilde{A}_{2}, \operatorname{Ker}\left(\widetilde{A}_{2}-\widetilde{A}_{1}\right)=\operatorname{dom} A, \\
& \left(\widetilde{A}_{1}-A_{\mu}\right):\left(\widetilde{A}_{2}-\widetilde{A}_{1}\right)=\left(A_{M}-\widetilde{A}_{2}\right):\left(\widetilde{A}_{2}-\widetilde{A}_{1}\right)=0,
\end{aligned}
$$

and such that $\widetilde{Q}_{1}(z)$ and $\widetilde{Q}_{2}(z)=-\widetilde{Q}_{1}^{-1}(z)$ have the operator representations (5.1). Moreover, $G=\left(\widetilde{A}_{2}-\widetilde{A}_{1}\right)^{1 / 2}$, see the proof of Proposition 6.1 . Since $\operatorname{ran} G^{\times}=\mathfrak{N}$, the conditions (6.1) are equivalent to

$$
\lim _{x \uparrow-1}\left(\left(\widetilde{A}_{1}-x I\right)^{-1} h, h\right)=+\infty \quad \text { and } \quad \lim _{x \downarrow 1}\left(\left(x I-\widetilde{A}_{2}\right)^{-1} h, h\right)=+\infty
$$

for every $h \in \mathfrak{N} \backslash\{0\}$. According to (2.2) these last two relations hold if and only if

$$
\operatorname{ran}\left(I+\widetilde{A}_{1}\right)^{1 / 2} \cap \mathfrak{N}=\operatorname{ran}\left(I-\widetilde{A}_{2}\right)^{1 / 2} \cap \mathfrak{N}=\{0\},
$$

i.e., $\left(I+\widetilde{A}_{1}\right)_{\mathfrak{N}}=\left(I-\widetilde{A}_{2}\right)_{\mathfrak{N}}=0$, cf. (2.11). In view of (3.9) this means that $\widetilde{A}_{1}=A_{\mu}$ and $\widetilde{A}_{2}=A_{M}$, or equivalently, that $\widetilde{Q}_{1}(z)$ is the $Q_{\mu}$-function (and $\widetilde{Q}_{2}(z)$ is the $Q_{M}$-function) of $A$ in the sense of $[26]$.

Corollary 6.3. Let $\widetilde{Q}_{1}(z) \in \mathfrak{S}_{\mu}(\mathfrak{N})$. Then the following conditions are equivalent:

(i) $\left(\operatorname{Im} \widetilde{Q}_{1}(z)\right)^{-1} \in \mathcal{L}(\mathfrak{N})$ for some (equivalently for every) $z \in \mathbb{C} \backslash \mathbb{R}$;

(ii) the operator $C:=\lim _{z \rightarrow \infty} z\left(I-\widetilde{Q}_{1}(z)\right)$ has bounded inverse;

(iii) $\widetilde{Q}_{1}(z)$ is a $Q_{\mu}$-function of some simple Hermitian contraction $A$ satisfying $\left(A_{M}-\right.$ $\left.A_{\mu}\right) \mathfrak{N}=\mathfrak{N}$ (i.e. $A_{\mu}$ and $A_{M}$ are transversal). 
Proof. The equivalence of (i) and (ii) follows from the operator representation of $\widetilde{Q}_{1}(z)$ in (5.1), which shows that $\operatorname{Im} Q(i)$ is invertible if and only if $C^{1 / 2}=G=\left(\widetilde{A}_{2}-\widetilde{A}_{1}\right)^{1 / 2} \uparrow \mathfrak{N}$ is invertible.

If (i) holds then the corresponding rigged Hilbert space reduces to $\mathfrak{N}_{+}^{\widetilde{Q}_{1}}=\mathfrak{N}=\mathfrak{N}_{-}^{\widetilde{Q}_{1}}$ and (iii) follows from Theorem 6.2. The reverse implication that (iii) implies (i) is clear.

Remark 6.4. Assuming the property (i) in Corollary 6.3 it has been shown in [17] that a function $\widetilde{Q}_{1} \in \mathbf{N}_{\mathfrak{N}}[-1,1]$ is a $Q_{\mu}$-function if and only if $s-\lim _{z \rightarrow \infty} \widetilde{Q}_{1}(z)=I, \lim _{z \uparrow-1}\left(\widetilde{Q}_{1}(z) h, h\right)=$ $+\infty$ for all $h \in \mathfrak{N} \backslash\{0\}$, and $s-\lim _{z \downarrow 1} \widetilde{Q}_{1}(z)=0$. The proof in [17] is based on the boundary triplet approach. Observe, that these limit conditions are used to define the class $\mathfrak{S}_{\mu}(\mathfrak{N})$ in Section 4.

\section{Applications to nONNEGative SELFADJoint EXtensions of NONNEGATive SYMMETRIC LINEAR RELATIONS}

In this section some consequences of the results concerning $s c$-extensions of Hermitian contractions are translated to nonnegative selfadjoint extensions of a closed nonnegative symmetric linear relation in a separable Hilbert space $\mathfrak{H}$. There is a one-to-one correspondence between closed nonnegative linear relations $S$ and (the graphs of) Hermitian contractions $A$ in $\mathfrak{H}$ via the Cayley transform

$$
S=\mathcal{C}(A)=\{\{(I+A) f,(I-A) f\}: f \in \operatorname{dom} A\},
$$

cf. [24], [16]. Observe, that mul $S=\operatorname{ker}(I+A)$. One can recover $A$ from $S$ by the same transform, when $\mathcal{C}$ is interpreted for linear relations as follows

$$
\operatorname{graph} A=\mathcal{C}(S):=\left\{\left\{f+f^{\prime}, f-f^{\prime}\right\}:\left\{f, f^{\prime}\right\} \in S\right\} .
$$

The transform $\mathcal{C}$ preserves some basic operations on linear relations, like inclusions, intersections, adjoints, and, for instance, $S$ is selfadjoint if and only if $A=\mathcal{C}(S)$ is selfadjoint. For a closed nonnegative linear relation $S$ in $\mathfrak{H}$ the Friedrichs and Krein-von Neumann extension $S_{F}$ and $S_{N}$ of $S$ are connected with the extreme extensions $A_{M}$ and $A_{\mu}$ of $A$ via $S_{F}=\mathcal{C}\left(A_{\mu}\right)$ and $S_{N}=\mathcal{C}\left(A_{M}\right)$. It follows from $(-A)_{\mu}=-A_{M}$ and $(-A)_{M}=-A_{\mu}$ (see (3.6)) that

$$
S_{N}=\left(\left(S^{-1}\right)_{F}\right)^{-1} \text { and } S_{F}=\left(\left(S^{-1}\right)_{N}\right)^{-1} \text {, }
$$

cf. $[16]$.

To an arbitrary nonnegative selfadjoint extension $\widetilde{S}$ of $S$ one can associate a unique closed sesquilinear form $\widetilde{S}[u, v]$ with the domain $\mathcal{D}[\widetilde{S}]$ along to lines of densely defined case treated in [23]. In fact, $\widetilde{S}[u, v]$ is the closure of the form

$$
\widetilde{S}[f, g]:=\left(f^{\prime}, g\right), \quad\left\{f, f^{\prime}\right\},\left\{g, g^{\prime}\right\} \in \widetilde{S},
$$

cf. [31], and moreover

$$
\mathcal{D}[\widetilde{S}]=\operatorname{dom} \widetilde{S}_{s}^{1 / 2}, \widetilde{S}[u, v]=\left(\widetilde{S}_{s}^{1 / 2} u, \widetilde{S}_{s}^{1 / 2} v\right), \quad u, v \in \mathcal{D}[\widetilde{S}],
$$

where $\widetilde{S}_{s}$ stands for the orthogonal operator part of $\widetilde{S}$, see [9]. The closed forms associated with the nonnegative selfadjoint extensions $\widetilde{S}, S_{F}$, and $S_{N}$ satisfy the same inequalities as in the densely case (see [24], [5]):

$$
S_{N} \leq \widetilde{S} \leq S_{F}, \quad \mathcal{D}\left[S_{F}\right] \subseteq \mathcal{D}[\widetilde{S}] \subseteq \mathcal{D}\left[S_{N}\right]
$$


cf. [8]. Observe, that the form $S_{F}[\cdot, \cdot]$ is a closed restriction of the form $\widetilde{S}[\cdot, \cdot]$ and in particular of the form $S_{N}[\cdot, \cdot]$. Moreover, it follows from (7.3) that the form $S_{N}^{-1}[\cdot, \cdot]$ is a closed restriction of the form $S_{F}^{-1}[\cdot, \cdot]$. Now, let $S_{F}$ and $S_{N}$ be disjoint, i.e., assume that $S_{F} \cap S_{N}=S$, in which case $S_{F} \neq S_{N}$. The following question arises from the main results in the previous sections: Does there exist another pair $\left\{\widetilde{S}_{1}, \widetilde{S}_{2}\right\}$ of nonnegative selfadjoint extensions of $S$ which has the same properties as the pair $\left\{S_{F}, S_{N}\right\}$ :

$$
\left\{\begin{array}{l}
\widetilde{S}_{1} \cap \widetilde{S}_{2}=S, \\
\text { the form } \widetilde{S}_{1}[\cdot, \cdot] \text { is a closed restriction of the form } \widetilde{S}_{2}[\cdot, \cdot] \\
\text { the form } \widetilde{S}_{2}^{-1}[\cdot, \cdot] \text { is a closed restriction of the form } \widetilde{S}_{1}^{-1}[\cdot, \cdot]
\end{array}\right.
$$

The solution to this problem is connected with the existence of $s c$-extensions $\widetilde{A}_{1} \leq \widetilde{A}_{2}$ of the Hermitian contraction $A=\mathcal{C}(S)$, $\operatorname{dom} A=\operatorname{ran}(I+S)$, defined by (7.2), such that the properties (4.1) are satisfies.

Lemma 7.1. Let $\widetilde{S}$ be a nonnegative selfadjoint linear relation and let $\widetilde{A}=\mathcal{C}(\widetilde{S})$ be its Cayley transform in (7.2). Then

$$
\begin{gathered}
\mathcal{D}[\widetilde{S}]=\operatorname{ran}(I+\widetilde{A})^{1 / 2} \\
\widetilde{S}[u, v]=-(u, v)+2\left((I+\widetilde{A})^{(-1 / 2)} u,(I+\widetilde{A})^{(-1 / 2)} v\right), \quad u, v \in \mathcal{D}[\widetilde{S}] .
\end{gathered}
$$

Proof. Since

$$
\widetilde{S}=\{\{(I+\widetilde{A}) h,(I-\widetilde{A}) h\}: h \in \mathfrak{H}\}
$$

with $f=(I+\widetilde{A}) h$ one obtains

$$
\begin{aligned}
& \widetilde{S}[f, f]=((I-\widetilde{A}) h,(I+\widetilde{A}) h)=-\|(I+\widetilde{A}) h\|^{2}+2\left\|(I+\widetilde{A})^{1 / 2} h\right\|^{2}= \\
& =-\|f\|^{2}+2\left\|(I+\widetilde{A})^{(-1 / 2)} f\right\|^{2} .
\end{aligned}
$$

Now the closure procedure leads to the statement.

Theorem 7.2. A pair of nonnegative selfadjoint extensions $\left\{\widetilde{S}_{1}, \widetilde{S}_{2}\right\}$ of $S$ satisfies the conditions (7.4) if and only if the pair $\left\{\widetilde{A}_{1}, \widetilde{A}_{2}\right\}=\left\{\mathcal{C}\left(\widetilde{S}_{1}\right), \mathcal{C}\left(\widetilde{S}_{2}\right)\right\}$ of sc-extensions of the Hermitian contraction $A=\mathcal{C}(S)$ satisfies the conditions (4.1).

Proof. By definition $\widetilde{A}_{1} \leq \widetilde{A}_{2}$ if and only if $\widetilde{S}_{1} \geq \widetilde{S}_{2}$. Observe, that here the last inequality is implied by (7.4). It is clear that the conditions $\widetilde{S}_{1} \cap \widetilde{S}_{2}=S$ and $\operatorname{ker}\left(\widetilde{A}_{2}-\widetilde{A}_{1}\right)=\operatorname{dom} A$ are equivalent. By Remark 4.1 the conditions $\left(\widetilde{A}_{2}-\widetilde{A}_{1}\right):\left(\widetilde{A}_{1}-A_{\mu}\right)=\left(\widetilde{A}_{2}-\widetilde{A}_{1}\right):\left(A_{M}-\widetilde{A}_{2}\right)=0$ can be rewritten as in (4.2). Now Lemma 2.8 shows that $I+\widetilde{A}_{1}=\left(I+\widetilde{A}_{2}\right)^{1 / 2} \widetilde{P}\left(I+\widetilde{A}_{2}\right)^{1 / 2}$, where $\widetilde{P}_{2}$ is an orthogonal projection in $\mathfrak{H}$. Hence, it follows from Lemma 2.11 that there is a partial isometry $U$ with the initial space $\overline{\operatorname{ran}}\left(I+\widetilde{A}_{1}\right)$, such that $\left(I+\widetilde{A}_{1}\right)^{1 / 2}=\left(I+\widetilde{A}_{2}\right)^{1 / 2} U$. This implies that $\left(I+\widetilde{A}_{2}\right)^{(-1 / 2)} g=U\left(I+\widetilde{A}_{1}\right)^{(-1 / 2)} g$ for all $g \in \operatorname{ran}\left(I+\widetilde{A}_{1}\right)^{1 / 2}$. Therefore, by Lemma 7.1 we get $\mathcal{D}\left[\widetilde{S}_{1}\right] \subseteq \mathcal{D}\left[\widetilde{S}_{2}\right]$ and $\widetilde{S}_{1}[u, v]=\widetilde{S}_{2}[u, v]$ for all $u, v \in \mathcal{D}\left[\widetilde{S}_{1}\right]$. So, the form $\widetilde{S}_{1}[\cdot, \cdot]$ is a closed restriction of the form $\widetilde{S}_{2}[\cdot, \cdot]$.

Similar arguments show that $I-\widetilde{A}_{2}=\left(I-\widetilde{A}_{1}\right)^{1 / 2} \widetilde{Q}\left(I-\widetilde{A}_{1}\right)^{1 / 2}$ for some orthogonal projection $\widetilde{Q}$ in $\mathfrak{H}$ and further that the form $\widetilde{S}_{2}^{-1}[\cdot, \cdot]$ is a closed restriction of the form $\widetilde{S}_{1}^{-1}[\cdot, \cdot]$. 
Converselly, let the form $\widetilde{S}_{1}[\cdot, \cdot]$ be a closed restriction of the form $\widetilde{S}_{2}[\cdot, \cdot]$. By Lemma 7.1 one has $\operatorname{ran}\left(I+\widetilde{A}_{1}\right)^{1 / 2} \subseteq \operatorname{ran}\left(I+\widetilde{A}_{2}\right)^{1 / 2}$ and $\left(I+\widetilde{A}_{2}\right)^{(-1 / 2)} f=W\left(I+\widetilde{A}_{1}\right)^{(-1 / 2)} f$ for all $f \in \operatorname{ran}\left(I+\widetilde{A}_{1}\right)^{1 / 2}$ with some partial isometry $W$ with the initial subspace $\overline{\operatorname{ran}}\left(I+\widetilde{A}_{1}\right)$. Hence, $\left(I+\widetilde{A}_{1}\right)^{1 / 2}=\left(I+\widetilde{A}_{2}\right)^{1 / 2} W$ and by Lemma $2.11\left(I+\widetilde{A}_{1}\right):\left(\widetilde{A}_{2}-\widetilde{A}_{1}\right)=0$. The last identity is equivalent to $\left(\widetilde{A}_{1}-A_{\mu}\right):\left(\widetilde{A}_{2}-\widetilde{A}_{1}\right)=0$, see Remark 4.1 .

Similarly, the condition that the form $\widetilde{S}_{2}^{-1}[\cdot, \cdot]$ is a closed restriction of the form $\widetilde{S}_{1}^{-1}[\cdot, \cdot]$ leads to the equality $\left(A_{M}-\widetilde{A}_{2}\right):\left(\widetilde{A}_{2}-\widetilde{A}_{1}\right)=0$.

The following result gives the answer to the problem posed above, cf. (7.4).

Corollary 7.3. Let $S$ be a closed nonnegative symmetric linear relation in a separable Hilbert space $\mathfrak{H}$ and let $S_{F} \cap S_{N}=S$. If the defect subspaces of $S$ are infinite-dimensional, then there are pairs $\left\{\widetilde{S}_{1}, \widetilde{S}_{2}\right\}$ of nonnegative selfadjoint extensions of $S$ which satisfy the properties (7.4), but which are different from the pair $\left\{S_{F}, S_{N}\right\}$. If the defect subspaces of $S$ are finite-dimensional, then every pair $\left\{\widetilde{S}_{1}, \widetilde{S}_{2}\right\}$ with the properties (7.4) coincides with the pair $\left\{S_{F}, S_{N}\right\}$.

\section{REFERENCES}

[1] W.N. Anderson, Jr., Shorted operators, SIAM J. Appl. Math. 20 (1971), 520-525.

[2] W.N. Anderson and R.J. Duffin, Series and parallel addition of matrices, J. Math. Anal. Appl. 26 (1969), 576-594.

[3] W.N. Anderson and G.E. Trapp, Shorted operators. II, SIAM J. Appl. Math. 28 (1975), 60-71.

[4] T. Ando, Lebesque-type decomposition of positive operators, Acta Sci. Math. (Szeged) 38 (1976), $253-260$.

[5] T. Ando and K. Nishio, Positive selfadjoint extensions of positive symmetric operators, Tohoku Math. J., 22 (1970), 65-75.

[6] Yu.M. Arlinskii, Contractive extensions of a dual pair of contractions and their resolvents, Ukrain. Math.Journ. 37, No.2 (1985), 247-250.

[7] Yu.M. Arlinskii, Theory of operator means, Ukr. Math. Journ. 42, No.6 (1990), 723-730.

[8] Yu.M. Arlinskii, Extremal extensions of sectorial linear relations, Matematychni Studii 7, No.1 (1997), 81-96.

[9] Yu.M. Arlinskiı̌, S. Hassi, Z. Sebestyén, and H.S.V. de Snoo, On the class of extremal extensions of a nonnegative operator, Oper. Theory Adv. Appl. 127 (2001), 41-81.

[10] Yu.M. Arlinskii and E.R. Tsekanovskiı̌, Quasi selfadjoint contractive extensions of Hermitian contractions, Teor. Funkts., Funkts. Anal. Prilozhen 50 (1988), 9-16.

[11] Gr. Arsene and A. Geondea, Completing matrix contractions, J. Oper. Theory 7 (1982), 179-189.

[12] Yu.M. Berezanskii, Expansions in eigenfunction of selfadjoint operators, Amer. Math. Soc., Providence, 1968.

[13] J.E. Brasche and H. Neidhardt, Has every symmetric operator a closed symmetric restriction whose square has trivial domain? Acta Sci.Math. (Szeged) 58 (1993), 425-430.

[14] M.S. Brodskii, Triangular and Jordan representations of linear operators, Nauka, Moscow 1968 (Russian).

[15] V. Cachia, H. Neidhardt, and V. Zagrebnov, Comments on the Trotter product formula error-bound estimates for nonselfadjoint semigroups, Int. Eq. Oper. Theory 42 (2002), 425-428.

[16] E.A. Coddington and H.S.V. de Snoo, Positive selfadjoint extensions of positive symmetric subspaces, Math. Z. 159 (1978), 203-214.

[17] V.A. Derkach and M.M. Malamud, The extension theory of hermitian operators and the moment problem, J. Math. Sciences, 73 (1995), 141-242.

[18] V.A. Derkach, S. Hassi, M.M. Malamud, and H.S.V. de Snoo, Generalized resolvents of symmetric operators and admissibility, Methods of Functional Analysis and Topology, 6 (2000), 24-55. 
[19] R.G. Douglas, On majorization, factorization and range inclusion of operators in Hilbert space, Proc. Amer. Math. Soc. 17 (1966), 413-416.

[20] S.L. Eriksson and H. Leutwiler, A potential theoretic approach to parallel addition, Math. Ann. 274 (1986), 301-317.

[21] P.A. Fillmore and J.P. Williams, On operator ranges. Advances in Math. 7 (1971), 254-281.

[22] I.S. Kac and M.G. KreĬn, $R$-functions - analytic functions mapping the upper halfplane into itself, Supplement to the Russian edition of F.V. Atkinson, Discrete and continuous boundary problems, Mir, Moscow 1968 (Russian) (English translation: Amer. Math. Soc. Transl. Ser. 2, 103 (1974), 1-18).

[23] T. Kato, Perturbation theory, Springer-Verlag, Berlin-Heidelberg-New York, 1966.

[24] M.G. Kreln, Theory of selfadjoint extensions of semibounded operators and its applications. I, Mat. Sb. 20 No.3 (1947), 431-498.

[25] M.G. Krĕn and H. Langer, Über die $Q$-function eines $\pi$-hermiteschen Operators im Raume $\Pi_{\kappa}$, Acta Sci. Math. (Szeged) 34 (1973), 191-230.

[26] M.G. Krel̆n and I.E. Ovcharenko, On $Q$-functions and sc-extensions of a Hermitian contraction with nondense domain, Sibirsk. Mat. Journ. 18 No.5 (1977), 1032-1056.

[27] F. Kubo and T. Ando, Means of positive linear operators, Math. Ann. 246 No.3 (1980), $205-224$.

[28] K. Nishio, T. Ando, Characterizations of operators derived from network connections, J. Math. Anal. Appl. 53 (1976), 539-549.

[29] E.L. Pekarev, Shorts of operators and some extremal problems. Acta Sci. Math. (Szeged) 56 (1992), 147-163.

[30] E.L. Pekarev and Yu.L. Shmulyan, Parallel addition and parallel subtraction of operators, Izv. Akad. Nauk SSSR, Ser Mat. 40 No.2 (1976), 366-387.

[31] F.S. Rofe-Beketov, Numerical range of linear relation and maximal linear relations, Functions Theory, Functional Anal. and their Appl. 44 (1985), 103-111. (Russian)

[32] B. Sz.-Nagy and C. Foias, Harmonic analysis of operators on Hilbert space, North-Holland, New York, 1970.

[33] K. Schmüdgen, On domains of powers of closed symmetric operators, J. Oper. Theory 9 No.1 (1983), 53-75. Correction in: J. Oper. Theory 12 No.1 (1984), 199.

[34] Yu.L. Shmul'yan, Hellinger's operator integral, Mat. Sb. 49 No.4 (1959), 381-430.

[35] Yu.L. Shmul'yan and R.N. Yanovskaya, Blocks of a contractive operator matrix, Izv. Vyssh. Uchebn. Zaved., Mat. No.7 (1981), 72-75.

Department of Mathematical Analysis, East Ukrainian National University, Kvartal Molodyozhny 20-A, Lugansk 91034, Ukraine

E-mail address: yma@snu.edu.ua

Department of Mathematics and Statistics, University of VaAsa, P.O. Box 700, 65101 VAASA, FINLAND

E-mail address: sha@uwasa.fi

Department of Mathematics, University of Groningen, Postbus 800, 9700 AV Groningen, NEDERLAND

E-mail address: desnoo@math.rug.nl 